\title{
Follow-up Imaging of Disk Candidates from the Disk Detective Citizen Science Project: New Discoveries and False Positives in WISE Circumstellar Disk Surveys
}

\author{
Steven M. Silverberg ${ }^{1,2}$ (iD), Marc J. Kuchner ${ }^{2}$ (D) , John P. Wisniewski ${ }^{1}$ (D), Alissa S. Bans ${ }^{3}$ (D), John H. Debes ${ }^{4}$ (D), Scott J. Kenyon ${ }^{5}$ (D) \\ Christoph Baranec ${ }^{6}$ (D), Reed Riddle ${ }^{7}$ (D) Nicholas Law ${ }^{8}$ (i), Johanna K. Teske ${ }^{9}$, Emily Burns-Kaurin ${ }^{10}$, Milton K. D. Bosch ${ }^{10}$ (iD, \\ Tadeas Cernohous ${ }^{10}$, Katharina Doll ${ }^{10}$ (iD), Hugo A. Durantini Luca ${ }^{10}$ (D), Michiharu Hyogo ${ }^{10}$ (iD), Joshua Hamilton ${ }^{10}$, \\ Johanna J. S. Finnemann ${ }^{10}$, Lily Lau ${ }^{10}$, \\ and \\ Disk Detective Collaboration \\ ${ }^{1}$ Homer L. Dodge Department of Physics and Astronomy, University of Oklahoma, 440 W. Brooks Street, Norman, OK 73019, USA; silverberg@ou.edu \\ ${ }^{2}$ NASA Goddard Space Flight Center, Exoplanets and Stellar Astrophysics Laboratory, Code 667, Greenbelt, MD 20771, USA \\ 3 Department of Physics, Emory University, 201 Dowman Drive, Atlanta, GA 30322, USA \\ ${ }_{5}^{4}$ Space Telescope Science Institute, 3700 San Martin Drive, Baltimore, MD 21218, USA \\ ${ }^{5}$ Smithsonian Astrophysical Observatory, 60 Garden Street, Cambridge, MA 02138, USA \\ ${ }^{6}$ Institute for Astronomy, University of Hawai' ${ }^{i}$ at Mānoa, Hilo, HI 96720-2700, USA \\ ${ }^{7}$ Division of Physics, Mathematics, and Astronomy, California Institute of Technology, Pasadena, CA 91125, USA \\ ${ }^{8}$ Department of Physics and Astronomy, University of North Carolina at Chapel Hill, Chapel Hill, NC 27599-3255, USA \\ ${ }^{9}$ Carnegie DTM, 5241 Broad Branch Road NW, Washington, DC 20015, USA \\ Received 2018 May 7; revised 2018 September 20; accepted 2018 September 21; published 2018 November 16
}

\begin{abstract}
The Disk Detective citizen science project aims to find new stars with excess $22 \mu \mathrm{m}$ emission from circumstellar dust in the AllWISE data release from the Wide-field Infrared Survey Explorer. We evaluated 261 Disk Detective objects of interest with imaging with the Robo-AO adaptive optics instrument on the $1.5 \mathrm{~m}$ telescope at Palomar Observatory and with RetroCam on the $2.5 \mathrm{~m}$ du Pont Telescope at Las Campanas Observatory to search for background objects at 0 " $15-12^{\prime \prime}$ separations from each target. Our analysis of these data leads us to reject $7 \%$ of targets. Combining this result with statistics from our online image classification efforts implies that at most $7.9 \% \pm 0.2 \%$ of AllWISE-selected infrared excesses are good disk candidates. Applying our false-positive rates to other surveys, we find that the infrared excess searches of McDonald et al. and Marton et al. all have false-positive rates $>70 \%$. Moreover, we find that all 13 disk candidates in Theissen \& West with W4 signal-to-noise ratio $>3$ are false positives. We present 244 disk candidates that have survived vetting by follow-up imaging. Of these, 213 are newly identified disk systems. Twelve of these are candidate members of comoving pairs based on Gaia astrometry, supporting the hypothesis that warm dust is associated with binary systems. We also note the discovery of $22 \mu \mathrm{m}$ excess around two known members of the Scorpius-Centaurus association, and we identify known disk host WISEA J164540.79-310226.6 as a likely Sco-Cen member. Thirty of these disk candidates are closer than $\sim 125 \mathrm{pc}$ (including 26 debris disks), making them good targets for both direct-imaging exoplanet searches.
\end{abstract}

Key words: catalogs - infrared: planetary systems - methods: data analysis - protoplanetary disks - surveys

Supporting material: figure set, machine-readable tables

\section{Introduction}

With higher sensitivity than any previous full-sky infrared survey instrument, the Wide-field Infrared Survey Explorer (WISE; Wright et al. 2010) detected over 747 million sources in its all-sky survey. Many teams have searched for new circumstellar disks in the WISE data based on infrared excess at W4 (22 $\mu \mathrm{m}$ emission) compared to $W 1$ (3.4 $\mu \mathrm{m}$ emission), discovering thousands of candidate debris disks (see, e.g., Kennedy \& Wyatt 2013; Wu et al. 2013; Patel et al. 2014; Cotten \& Song 2016; Patel et al. 2017) and young stellar object (YSO) disks (Liu et al. 2011, 2014; Rebull et al. 2011; Koenig \& Leisawitz 2014; Koenig et al. 2015). In particular, because of its sensitivity and full-sky scope, the WISE mission is uniquely suited to the search for M-dwarf debris disks, which are of particular interest because of the relative lack of detected disks around these stars in comparison to higher-mass stars (e.g., Plavchan et al. 2005, 2009; Lestrade et al. 2009;

\footnotetext{
${ }^{10}$ Disk Detective Advanced User Team.
}

Avenhaus et al. 2012; Morey \& Lestrade 2014; Theissen \& West 2014; Binks \& Jeffries 2017).

However, confusion and contamination limit every search for disks with WISE. The point-spread function (PSF) at W4 has an FWHM of $12^{\prime \prime}$. This wide PSF can allow emission from multiple point sources (e.g., background stars) or image artifacts to contribute to the $W 4$ photometry, producing a false-positive [W1]-[W4] excess. Additionally, the color loci of debris disks and YSOs overlap the color loci of several other astronomical phenomena, including background galaxies and stars embedded in nebulosity (Koenig et al. 2012). To eliminate these false positives, objects must be examined in visible and near-infrared images along with the WISE images. Most published searches have utilized visual inspection of the WISE images (see, e.g., Debes et al. 2011; Kennedy \& Wyatt 2012; Wu et al. 2013; Cruz-Saenz de Miera et al. 2014; Patel et al. 2014) to address these contamination and confusion problems.

Kennedy \& Wyatt (2012) produced a study of these confusion and contamination issues, focusing on infrared 
excesses around stars observed by Kepler (Borucki et al. 2010), with the goal of expanding the number of known stars that host both planets and debris disks. They searched for infrared excesses in a cross-match of the Kepler Input Catalog (KIC) to 2MASS and WISE, finding 7965 disk candidates. However, they argued that all but $271(3.4 \%)$ of these objects were coincident with Galactic dust emission, as identified by the IRAS $100 \mu \mathrm{m}$ background, and therefore were false positives.

The Disk Detective citizen science/crowd-sourcing project (Kuchner et al. 2016, hereafter Paper I) uses citizen science to examine infrared excess candidates from WISE, beginning with a website, http://www.diskdetective.org, where volunteers examine images from WISE, the Two-Micron All-Sky Survey (2MASS), the Digitized Sky Survey (DSS), and the Sloan Digital Sky Survey (SDSS) to check for false positives. Since launch in 2014 January, over 30,000 users have made over 2.6 million classifications via this Zooniverse website. Shortly after project launch, a group of highly dedicated volunteers began their own email discussion group for the project. Since then, members of this "advanced user group" have helped train other users and research follow-up targets in the literature, and they now form a crucial extension of the Disk Detective science team (Paper I).

In this paper, we address two specific forms of false positive that occur in the Disk Detective input catalog and other searches for circumstellar disks with WISE:

1. Confusion in the WISE images: the contribution to noise in an image due to superposed signals from faint unresolved sources that cluster on the scale of the observing beam.

(a) We use data from the DSS, the SDSS, and the 2MASS to search for background sources via the Disk Detective website.

(b) We use high-resolution imaging on small telescopes (the $1.5 \mathrm{~m}$ Telescope at Mt. Palomar and the du Pont Telescope at Las Campanas) to identify background sources that are too faint for SDSS and 2MASS or unresolved by these surveys.

2. Contamination of the WISE images: the presence of image artifacts (e.g., diffraction spikes, latent images, or optical ghosts) within the WISE beam. We search for these contaminants by examining the WISE images via the Disk Detective website.

We present the first results from our follow-up imaging campaigns with the Robo-AO adaptive optics instrument on the $1.5 \mathrm{~m}$ telescope at Palomar Observatory (Baranec et al. 2014) and the RetroCam instrument on the du Pont Telescope at Las Campanas Observatory in Chile (e.g., Rheault et al. 2014). These instruments provided an angular resolution of 0 ". 15 and $<1^{\prime \prime}$, respectively, improving on the $5^{\prime \prime}$ effective resolution of the 2MASS Point Source Catalog. ${ }^{11}$ We combine these observations with the results of the website-based evaluation to estimate the fraction of WISE excesses that are true disk candidates. In Section 2, we review our website classification procedure, analyze the distribution of clean sources and false positives, and consider the results of an advanced-user-driven literature review. In Section 3, we review our procedure for selecting these particular targets for further examination, and we discuss our methodology for collecting high-resolution

\footnotetext{
11 As listed here: https://www.ipac.caltech.edu/2mass/releases/allsky/doc/ sec2_2a.html.
}

images of these targets. In Section 4, we describe our method for identification of contaminated targets. In Section 5, we present the results of our follow-up observations, estimate the presence of further unresolved sources, and combine these with the results from website analysis to estimate the ultimate yield of the Disk Detective input catalog. We apply these findings to other surveys in Section 6, including a reanalysis of the M-dwarf disk search of Theissen \& West (2014). In Section 7, we present our list of uncontaminated disk candidates. Finally, in Section 8 we summarize our results and discuss future plans for these targets and high-resolution follow-up imaging.

\section{Rejection of False Positives via the DiskDetective.org Website and Literature Review}

The online engine of our citizen science disk search is DiskDetective.org, where users view sets of images showing the same WISE point source in several bands. In this section, we review our online classification method (discussed in more depth in Paper I) and analyze the latest online classification results.

\subsection{DiskDetective.org: Identification of WISE Debris Disk Candidates with Citizen Science}

The selection of our input catalog is detailed in Table 1 of Paper I. Briefly, we selected objects from the AllWISE Source Catalog with significant $[W 1]-[W 4]$ excess $([W 1]-[W 4]>$ $\left.0.25,[W 1]-[W 4]>5 \sigma_{[W 1]-[W 4]}\right)$ and high signal-to-noise ratio $(\mathrm{S} / \mathrm{N}>10)$ at $W 4$ that were not flagged as being contaminated in any way as part of the AllWISE source processing. These objects become "subjects" as part of our input catalog. ${ }^{12}$ For each subject, we generated a "flip book" of 9-15 1-arcminsquare images of the subject from the DSS, the seventh data release of the Sloan Digital Sky Survey (SDSS DR7), 2MASS, and AllWISE. Images were overlaid with a red circle of radius 10.5 arcsec, the area that must be clear of contamination for the AllWISE photometry to be trustworthy, and small crosshairs indicating the center of the $W 1$ source. Users view each flip book as an animation or scroll through frame-by-frame using a scrub bar. Users then choose from six classification buttons, labeled "Multiple Objects in the Red Circle," "Object Moves off the Crosshairs," "Extended beyond Circle in WISE Images," "Empty Circle in WISE Images," "Not Round in DSS2 or 2MASS Images," and "None of the Above/Good Candidate." Users can choose either "None of the Above" or as many of the other descriptions as apply to a target. This classification method robustly identifies type-1a and type- 2 false positives, as described above.

In this paper, we focus only on subjects that have been retired from active classification, which we refer to as "complete." In Paper I, we defined this cutoff as 15 classifications. After the publication of Paper I, we put into place a new retirement scheme and corrected some objects for saturation effects at $W 1$. The new retirement scheme is described in Appendix A.1, and our correction for $W 1$ saturation is treated in Appendix A.2. As of 2018 January 5, $62 \%$ of subjects were complete, providing the large sample of 149,273 subjects we analyze here.

We define a "good" subject as one where the majority of classifiers of a subject label it "None of the Above/Good

\footnotetext{
12 The full input catalog is available via the MAST archive: https://mast. stsci.edu.
} 

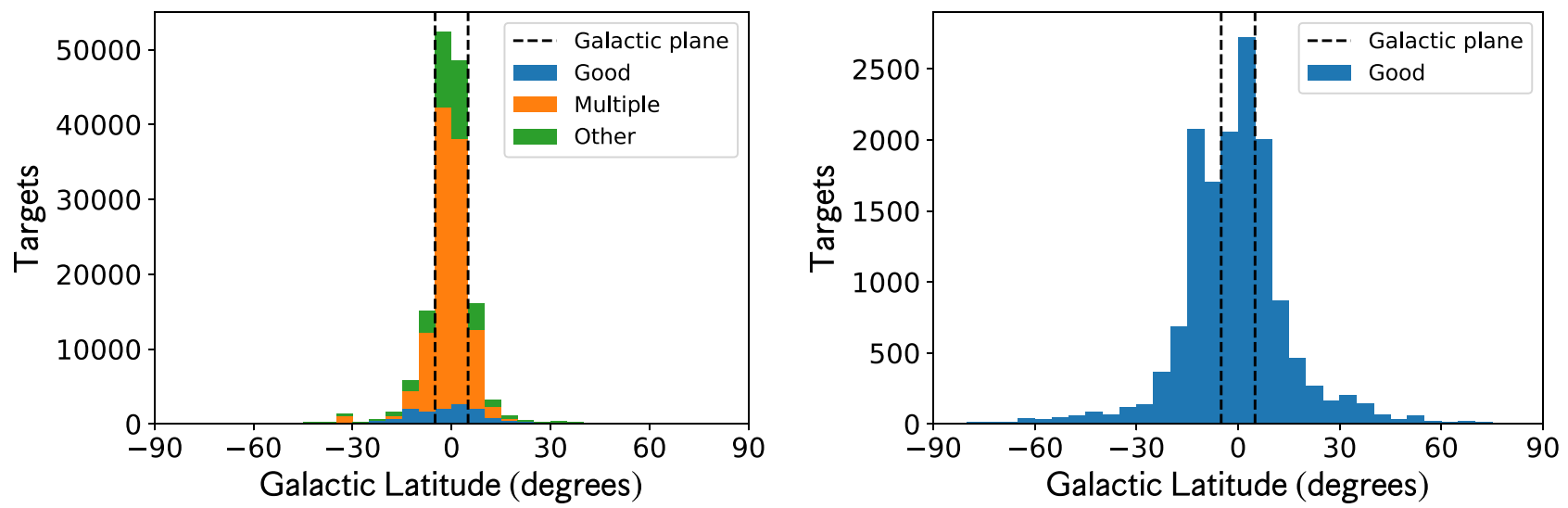

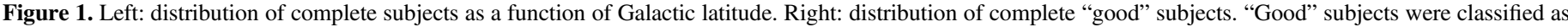

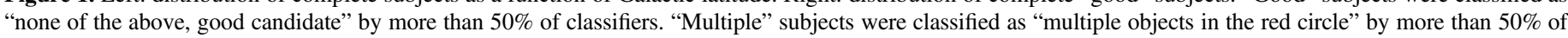
classifiers. All other complete subjects were labeled "other."

Object." We refer to a subject as "multiple" if a majority of classifiers label that subject as having "Multiple Objects in the Red Circle"; these "multiple" subjects are the dominant false positive rejected by our volunteers. We choose this definition (based on a majority of classifiers rather than a majority of votes) because users can select more than one option with each classification; this metric shows us subjects that most users agree have "multiple objects in the red circle," even if they also have other flaws. For convenience, we refer to all subjects that do not meet these definitions of "good" or "multiple" as "other" in this paper.

\subsection{False-positive Rates in Website Classifications}

Figure 1 shows the distribution of raw numbers of complete subjects broken down by category ("good," "multiple," or "other") as a function of Galactic latitude and the distribution of "good" objects alone as a function of Galactic latitude. Most of the complete subjects are false positives; only $9.80 \%$ $( \pm 0.08 \%)$ of the complete subjects are labeled "good" by a majority of volunteers. "Multiples" are the dominant form of false positive: they make up $68.87 \%( \pm 0.12 \%)$ of all complete subjects.

Figure 2 shows the rate of multiples as a function of Galactic latitude. "Multiples" dominate especially in the Galactic plane; $74.84 \%( \pm 0.16 \%)$ of subjects in the $-5^{\circ}<b<5^{\circ}$ range were classified as multiples, while only $4.74 \%( \pm 0.30 \%)$ of subjects in that range are good, with over 100,000 complete subjects in this range. We also observe a large spike in the "multiple" rate in Galactic latitudes $-35^{\circ}<b<-30^{\circ}$. Figure 3 shows the density of "multiples" (i.e., the fraction of subjects that are multiples) as a function of Galactic latitude and longitude (redder indicates a larger fraction); this plot reveals that the spike in the $-35^{\circ}<b<-30^{\circ}$ range in Figure 2 is associated with the LMC. Figure 3 also shows a higher density of "multiples" in the SMC than in its surroundings. Our statistics are poor in the $60^{\circ}<b<90^{\circ}$ range because there are fewer complete subjects in this region of sky, which only recently became active on the website.

\subsection{Literature Review by Citizen Scientists}

The next stage of our process is to review the published literature on each of the good objects; discard point-source false positives like known $\mathrm{M}$ giants, classical Be stars, and

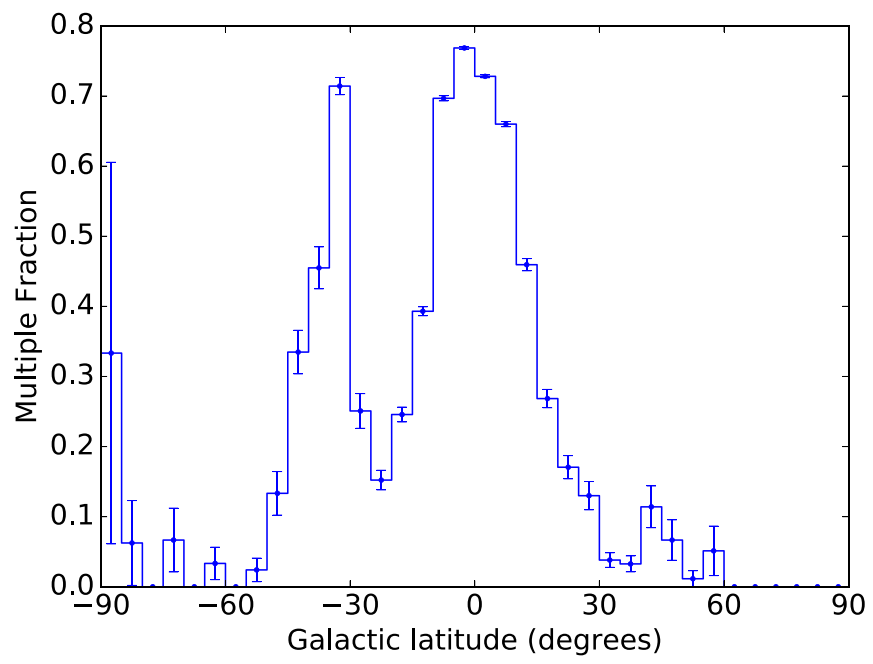

Figure 2. Rate of "multiple" subjects as a fraction of all complete subjects as a function of Galactic latitude. "Multiple" subjects are most common in the Galactic plane and in the Large Magellanic Cloud (LMC).

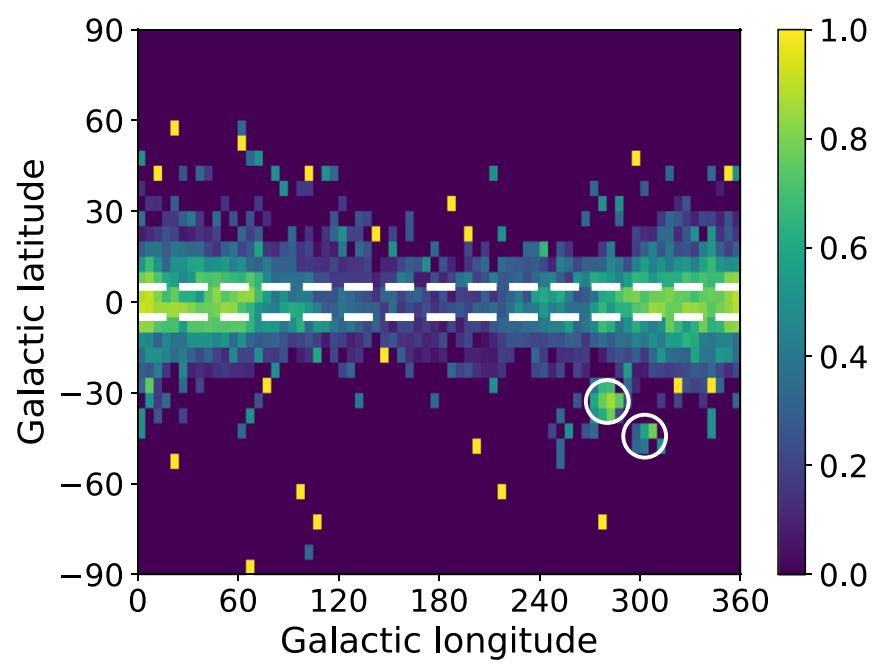

Figure 3. Heat map showing the fraction of complete objects in each $5^{\circ} \times 5^{\circ}$ bin that are "multiple." Redder colors indicate where a larger fraction of the total has been classified as "multiple." Dark blue indicates that a bin contains no multiples. Multiples are more prevalent in the plane (between white dashed lines) of the Galaxy and toward the Galactic center. Outside of the Galactic plane, a higher density of "multiples" is observed in the Large and Small Magellanic Clouds (locations indicated by the white circles). 
Table 1

Summary of Observations with Robo-AO

\begin{tabular}{lc}
\hline \hline UT Date & Objects observed \\
\hline 2014 Jun 14 & 15 \\
2014 Jun 15 & 12 \\
2014 Jun 16 & 2 \\
2014 Jun 19 & 1 \\
2014 Jul 12 & 1 \\
2014 Jul 13 & 18 \\
2014 Jul 14 & 1 \\
2014 Jul 16 & 1 \\
2014 Jul 17 & 6 \\
2014 Jul 18 & 1 \\
2014 Aug 21 & 2 \\
2014 Aug 29 & 2 \\
2014 Aug 30 & 4 \\
2014 Aug 31 & 2 \\
2014 Sep 01 & 65 \\
2014 Sep 02 & 3 \\
2014 Sep 03 & 1 \\
2014 Nov 06 & 1 \\
2014 Nov 09 & 18 \\
2014 Nov 10 & 1 \\
2014 Nov 11 015 Mar 08 & 1 \\
2015 Mar 09 & 9 \\
\hline
\end{tabular}

active galactic nuclei (AGNs); and note rediscoveries of wellstudied disk systems. While this task began as a science team function, we have since trained members of the advanced user group to use SIMBAD and VizieR themselves to perform some of this work, as detailed in Paper I. These citizen scientists also reexamine the website flip books for these subjects as an additional check on the website classification. We require a minimum of two opinions on each object before a good candidate can become a Disk Detective Object of Interest (DDOI), an object worthy of additional follow-up observations. We decided that any previously known, well-studied disk systems (e.g., systems observed by the Spitzer Space Telescope or with coronagraphic disk images) should not be designated as DDOIs. We decided that our observing resources were better used on confirming newly detected excesses rather than following up well-studied disks; however, these systems are still valid disk detections, so we do not eliminate them as false positives.

So far, the advanced user team has reviewed 1465 good subjects. Of these, 1011 have become DDOIs, while an additional 252 subjects have not become DDOIs because they are known well-studied disk systems, for a false-positive rate from the literature review of $14 \%$.

\section{Follow-up Target Selection and Observations}

We have performed follow-up imaging of a subset of our DDOIs using the Robo-AO on the $1.5 \mathrm{~m}$ telescope at Mount Palomar and the RetroCam instrument on the Irenée R. du Pont Telescope at Las Campanas Observatory. Here we describe our target selection, observations, and reduction methodology for each telescope.

\subsection{Robo-AO Observations}

We observed 230 targets in the Sloan- $i$ filter (Baranec et al. 2014) with adaptive optics using Robo-AO. Dates of
Table 2

Summary of Observations with RetroCam/du Pont

\begin{tabular}{lccc}
\hline \hline UT Date & $\begin{array}{c}\text { Objects } \\
\text { observed }\end{array}$ & $\begin{array}{c}\text { Minimum total } \\
\text { integration time }\end{array}$ & $\begin{array}{c}\text { Maximum total } \\
\text { integration time }\end{array}$ \\
\hline 2015 Jun 30 & 4 & 25 & 25 \\
2015 Jul 01 & 52 & 25 & 100 \\
2015 Jul 02 & 27 & 25 & 100 \\
2015 Oct 26 & 19 & 25 & 100 \\
2015 Oct 27 & 39 & 25 & 100 \\
2015 Oct 28 & 40 & 25 & 100 \\
\hline
\end{tabular}

observations and number of targets observed on each date are listed in Table 1. Targets were selected for visibility from Mt. Palomar, 2MASS $J<14.5$ and $i<17$, the limiting magnitude of the telescope. Targets were observed as a sequence of fullframe-transfer detector readouts at the maximum rate of $8.6 \mathrm{~Hz}$ for $90 \mathrm{~s}$ of total integration. We corrected the individual images for detector bias and flat-fielding effects, and then combined them using post facto shift-and-add processing, using the target star as the tip-tilt star with $100 \%$ frame selection to synthesize a long-exposure image. Additionally, we synthesized shorterexposure images by selecting smaller percentages of frames based on quality, as in lucky imaging. In most cases, these yielded an inner working angle for detecting background objects of $\sim 0$." 15 , far less than the $6^{\prime \prime}$ of the $W 4$ half-width at half-maximum (HWHM).

\section{2. du Pont/RetroCam}

We also collected high-resolution images of 166 targets (including 15 observed with Robo-AO) in the Yc $\left(\lambda_{c}=\right.$ $1.035 \mu \mathrm{m})$ and Hc $\left(\lambda_{c}=1.621 \mu \mathrm{m}\right)$ filters using RetroCam on the 100 inch $(2.54 \mathrm{~m})$ Ireneé R. du Pont Telescope at Las Campanas Observatory. Dates of observations and number of targets observed on each date are listed in Table 2. Targets were selected for visibility from Las Campanas and primarily for 2MASS $J<14.5$. We observed targets using a five-point "dice" dither pattern. Individual images were corrected for dark current and flat-fielded using the difference between lamp-on and lamp-off dome flats. $H$-band sky images were generated for each target by median-combining images in a dither sequence without aligning them, removing stellar contributions via sigma clipping. The $H$-band science images were then sky-subtracted, using the sky image for that target. Once images were completely reduced (dark, flat, and sky corrected), images for each target in each band were then combined with the "imalign" and "imcombine" procedures in IRAF, using bright stars in the field of view (including the target star) to align the image stack. Seeing in these observations (as measured by the FWHM of a star other than the target in the stacked image) was generally $\sim 0$ !" 8 , still smaller than the W4 HWHM.

\section{Image Analysis}

A group of 10 citizen scientists examined the Robo-AO data with the SAOImage DS 9 software package to visually examine the data to identify images with faint background objects, providing them with a set of images analyzed by the science team as a training set. We developed the following method for qualitative analysis: 
1. Display the image with $100 \%$ frame selection as black/ white gray scale, $\min / \max$, and linear scaling to identify the target star.

2. Shift to "zscale" to look for fainter objects. This allows fainter background objects to emerge more clearly than they would otherwise.

3. Identify any faint background objects in the field of view, noting their positions.

4. Note which objects have background objects within $12 \operatorname{arcsec}$ of the target star.

Each volunteer independently analyzed a subset of the images using the above method. The group then discussed each image together to reach a consensus on each target. We followed a similar procedure with the du Pont data.

This visual inspection identified targets with evidence of background sources that could produce a false-positive excess. We then quantified whether these background sources significantly affected the excess at W4. To estimate the contribution each background object made at $W 4$, we determined magnitudes for the background objects with aperture photometry using the IRAF DAOphot package. We assumed that background objects exhibited either an M-dwarf spectral energy distribution (SED) or a power-law SED with spectral index zero (corresponding to a YSO or heavily reddened early-type star) and determined the colors of these objects (calculated in Appendix B and presented in Table 8). Using the recorded photometry and these colors, we estimated the flux of the background object at $W 4$ and subtracted this flux from the total $W 4$ flux to yield the intrinsic flux of the target itself (including any contribution from circumstellar material). We then recalculated the target's [W1-W4] color using this corrected $W 4$ flux to determine if a significant excess remained. The results are described in Section 5.

\section{False-positive Rates of AllWISE Disk Detections}

In this section, we determine false-positive rates based on the follow-up observations and unresolvable IR-bright background objects. We then combine these with the classification and literature review data to determine the overall false-positive rate for Disk Detective thus far, from which we estimate the eventual final yield of disks from AllWISE.

\subsection{False-positive Rates from High-resolution Follow-up}

The results of quantitative analysis of the 261 targets with reliable photometry observed with Palomar/Robo-AO and du Pont/Retrocam are presented in Figure 4. We combine the two samples without adjustment because (as described in Appendix B) both instruments are similarly sensitive to the same background objects. Overall, 244 of these 261 targets retain a significant infrared excess after the contribution of background objects has been removed, for a false-positive rate of $7 \% \pm 1 \%$. There is no detectable significant difference in contamination rate in the Galactic plane, due to the relatively small numbers involved (compared to the overall Disk Detective input catalog). Of the 39 objects in the $-5^{\circ}<$ $b<5^{\circ}$ range, three are contaminated, leaving a false-positive rate of $8 \%( \pm 4 \%)$. Out of the plane, 14 of 222 targets are contaminated, leaving a false-positive rate of $6 \%( \pm 2 \%)$.

We identified 16 objects as having insufficient excess at $W 4$ once the estimated contributions from background objects were

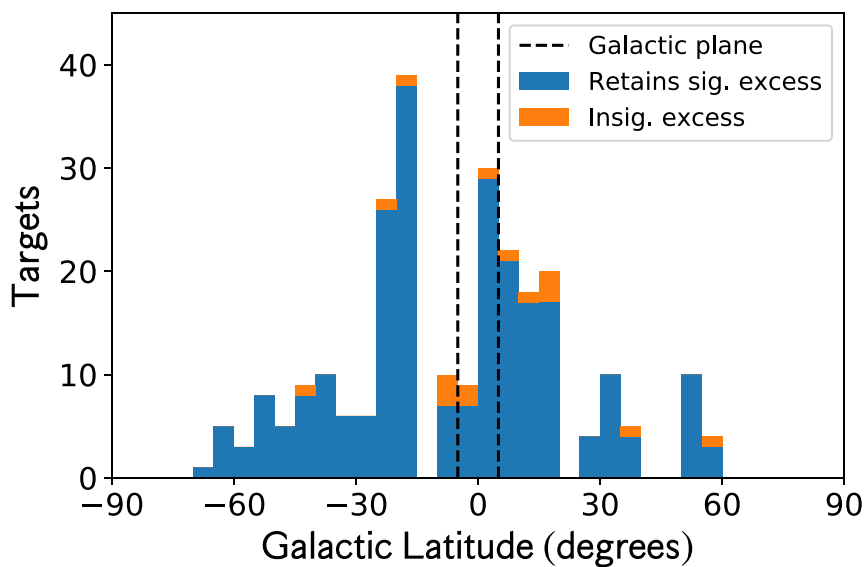

Figure 4. Objects with high-resolution imaging from Robo-AO and du Pont as a function of Galactic latitude. The overall false-positive rates are low in comparison to the rates from our website-based analysis. The rates inside and outside the Galactic plane show no significant difference.

Table 3

Targets with False-positive Excesses Due to Background Objects in Highresolution Follow-up Observations

\begin{tabular}{lcr}
\hline \hline & Identifiers & $\begin{array}{r}\text { Previous } \\
\text { citations }\end{array}$ \\
\hline Zooniverse & J000308.37+424452.4 & 1 \\
\hline AWI0005w52 & J010722.60+380143.9 & \\
AWI0005yiz & J020206.67+601741.4 & \\
AWI0002vbd & J021532.17+591424.4 & 1 \\
AWI0003cs7 & J032853.67+490412.8 & \\
AWI0004nfu & $\mathrm{J} 153046.05+342756.4$ & \\
AWI0000uj2 & $\mathrm{J} 161808.08+104551.4$ & \\
AWI00006nb & $\mathrm{J} 172912.43+005605.7$ & \\
AWI0005bk2 & $\mathrm{J} 181949.03+310841.7$ & \\
AWI0005brq & $\mathrm{J} 184141.31+313703.4$ & \\
AWI0005bud & $\mathrm{J} 193040.05+350609.9$ & \\
AWI0005lx9 & $\mathrm{J} 204443.79+425654.9$ & \\
AWI00055by & $\mathrm{J} 212959.78+413037.3$ & \\
AWI0005vyx & $\mathrm{J} 215305.45+682955.0$ & \\
AWI0005a9r & $\mathrm{J} 220503.97+444543.7$ & \\
AWI0005w1h & $\mathrm{J} 220601.14-020343.2$ & \\
AWI0000kk6 & & \\
\hline
\end{tabular}

References. (1) Paper I. (2) Wu et al. (2013). (3) Cotten \& Song (2016). (4) Clarke et al. (2005).

removed. We list these objects in Table 3, as well as any previous identifications as excess targets.

\subsection{False-positive Excess from Unresolvable Infrared Galaxies}

While the Robo-AO and RetroCam observations catch many otherwise-unresolved background sources, they leave one potential source of confusion unexplored: objects clustered on the scale of the $W 4$ beam with no counterpart in near-IR or red-optical light, such as luminous or ultraluminous infrared background galaxies, or (U)LIRGS (Papovich et al. 2004). While WISE is not well suited to exploring the density of these phenomena, previous higher-resolution mid-infrared surveys, operating at similar wavelengths, can provide constraints. Papovich et al. (2004) used Spitzer/MIPS data to identify a previously undetected population of infrared-luminous galaxies, quoting a cumulative distribution of number of galaxies as a function of source brightness at $24 \mu \mathrm{m}$. We can 
use this distribution to estimate the confusion noise from these galaxies in our $22 \mu \mathrm{m}$ data if we correct for sources that would be detected in website classifications and follow-up imaging.

We determined the minimum flux at $W 4$ for a background galaxy to produce a false-positive excess, assuming a bare stellar photosphere in the Rayleigh-Jeans tail. We also estimated the flux at $W 4$ at which a background galaxy would produce a visible signal in 2MASS $H$ images, assuming that such an object would be visible if its 2MASS $H$ flux were half that of the target. We applied these numbers to every good subject, finding the cumulative number of sources per steradian bright enough to produce a false positive, and subtracting from that the cumulative number of sources per steradian bright enough to have been detected as a background object in shorter-wavelength data. We then multiplied by the angular beam width at $W 4$ to get the expected number of sources. We treated this as a probability of the number of sources in the beam for each object, summing the probability for each object to estimate the number of good objects for which a background galaxy was the source of the false positive.

Of the 14,681 complete, good subjects, we expect that $7.4 \pm 2.7$ subjects, or $0.05 \% \pm 0.02 \%$, are contaminated by an unresolved background infrared-luminous galaxy, a negligible contribution.

\subsection{Expected Total Number of Disks in AllWISE}

Combining the data from user classifications, advanced user review, high-resolution follow-up observations, and background galaxy count estimates, we find that of the 149,273 complete subjects on the Disk Detective website, $7.9 \%$ $( \pm 0.2 \%)$ are likely to be stars with circumstellar material. Some $90.20 \%$ of subjects are eliminated by website evaluation, $1.35 \%$ were eliminated by literature review, $0.52 \%$ were eliminated by follow-up observations, and a near-negligible fraction $(<0.01 \%)$ are expected to have a false positive due to undetectable background galaxies.

Applying this number to the full brightness-limited sample currently on the website, we would expect to find $\sim 21,600$ disk candidates overall, out of 272,022 subjects in the brightnesslimited input catalog. Given that this analysis does not incorporate false positives that are only identifiable in spectroscopic follow-up (e.g., M giants, classical Be stars), we expect that this number is an upper limit to the number of debris and YSO disks with W4 excess in the AllWISE catalog. This $8.0 \%$ figure is higher than the $3.4 \%$ found by Kennedy \& Wyatt (2012). We attribute some of this difference to our full-sky scope, as Kennedy \& Wyatt (2012) only considered sources in or near the Galactic plane. We also hypothesize that some of the difference can be attributed to our detailed visual inspection of each source, rather than adopting a simple across-the-board cut based on $100 \mu \mathrm{m}$ flux, as they did.

\section{Application to Other WISE Disk Searches}

Given its large input catalog compared to other surveys, Disk Detective is well positioned to not only identify new warm debris disks in the WISE catalog, but also to inspect and reevaluate disks identified by other researchers. In this section, we estimate the numbers of published disks from other searches that are likely false positives, and we present a visual reinspection of the M-dwarf disk sample presented by Theissen \& West (2014).

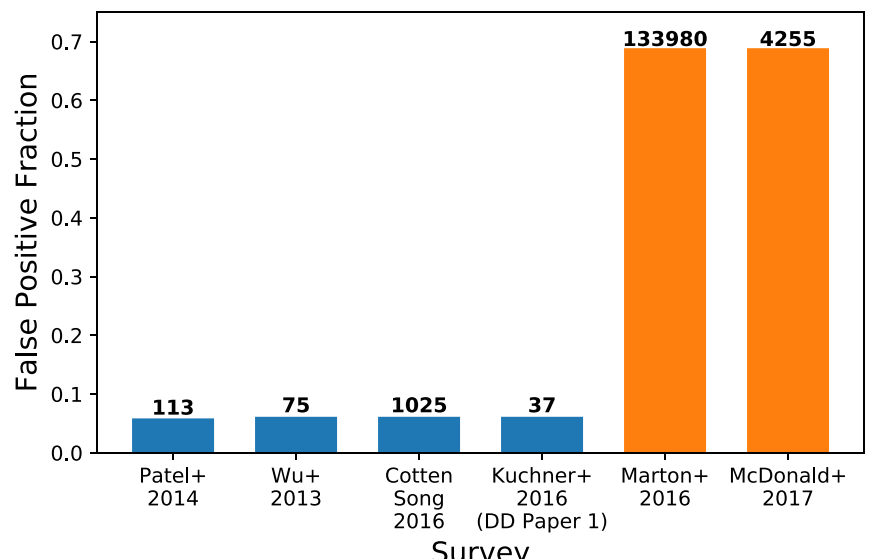

Figure 5. Expected false-positive fractions for published WISE disk searches. Searches that incorporate visual inspection of images (blue bars) have lower expected false-positive fractions than those that do not incorporate visual inspection (orange bars). Numbers above each bar indicate the number of disk candidates in each search.

\subsection{False Positives in Previous WISE Disk Searches}

Because our sample encompasses the full 2MASS/WISE cross-match, we can apply our false-positive rates to other searches for warm debris disks with WISE. While we cannot assess individual objects in other surveys, due to limited overlap with our follow-up, we estimate the number of disks in each survey likely to be a false positive based on the rates we determined, depicted in Figure 5.

1. Patel et al. (2014). This survey specifically avoided the Galactic plane in its cross-match with the Hipparcos catalog (van Leeuwen 2007), so we similarly apply an out-of-plane contamination rate. We will assume that the visual inspection described by the authors is comparable to the inspection and literature review by the Disk Detective team and will thus only apply the rate of contamination from follow-up imaging. Of the 113 new warm disks that were detected in this survey (Patel et al. 2015), we expect about seven to have previously undetected background objects that would contribute to a false-positive excess detection.

2. Wu et al. (2013). This survey did not specifically avoid the Galactic plane as a selection criterion in its crossmatch with Hipparcos, so we apply the statistics for the full sample to this paper's sample of 75 previously unidentified main-sequence stars with infrared excess indicative of a disk. We would therefore expect about five of these targets to be contaminated by one or more otherwise-undetected background objects.

3. Cotten \& Song (2016). This search identified $\sim 1750$ debris disk candidates from a thorough review of preWISE disk literature and a cross-match of AllWISE with the Tycho-2 (Høg et al. 2000) survey, of which 1025 are new excess detections. Applying our recovery rate for high-resolution follow-up to this sample, we would expect that $\sim 64$ targets would be contaminated by background objects only recoverable in high-resolution images that could produce a false-positive excess detection.

4. McDonald et al. (2012, 2017). These searches crossmatched photometry data from Hipparcos, Tycho, and various other catalogs, using astrometry from, respectively, 
Tycho and Gaia. These works then used SED fitting of the cross-matched data to estimate stellar and excess parameters for $>100,000$ objects. However, neither search considered the source of the excess (e.g., self-produced dust around AGB stars rather than debris disks), and neither search included a robust visual inspection of the actual images (which eliminates $\approx 90 \%$ of excess candidates in the Disk Detective search), relying instead on visual inspection of the fit SEDs. Because these searches select for objects with starlike SEDs rather than preselecting sources with infrared excess the way our search does, we cannot directly apply our overall false-positive rates to their search. However, since we estimate there to be $\sim 21,600$ disk candidates in AllWISE, we expect that $>79,000$ of the excesses identified by McDonald et al. $(2012,2017)$ are false positives.

5. Marton et al. (2016). This search used support vector machines (SVMs), a class of supervised learning algorithm, to identify 133,980 YSO candidates in AllWISE, by identifying objects that were a given type of false positive in the data via SIMBAD and using these to train the SVM to avoid such objects. However, there was no visual inspection of the images, sources were not preselected for excess, the W4 PSF was not taken into account, and there is no acknowledgment of multiples, which make up the bulk of our false positives. If we assume that the SVM algorithm identifies nonmultiple false positives as effective as Disk Detective, we also assume that literature review would eliminate the SIMBAD-identified non-YSOs in their final sample (as per Table 1 of that paper), and we further assume that a similar number of objects would be false positives in follow-up imaging, this survey would have a lower limit false positive rate of $74 \%$. While a search through WISE using machine-learning algorithms could prove valuable, the number of false positives that are only identified by visual inspection of images suggests that any such system would need to take the images themselves into account, rather than strictly learning based on photometric points and data quality flags.

6. Kuchner et al. (2016). As Paper I was published before analysis of Robo-AO and du Pont photometry was complete, we also apply our rate analysis to this paper. Of the 37 new disk candidates presented in that paper, we expect that about two would be contaminated.

The targets WISEA J153046.05+342756.4 (AWI0000uj2), WISEA J010722.60+380143.9 (AWI0005yiz), WISEA J032853.67+490412.8 (AWI0004nfu), and WISEA J215305.45 +682955.0 (AWI0005a9r) present salient examples of the importance of imaging follow-up. AWI0000uj2, an A0 star, appears in Wu et al. (2013), in Cotten \& Song (2016) as a "Reserved" excess candidate, and in Paper I. AWI0004nfu appears in Paper I and Zuckerman et al. (2012). AWI0005yiz and AWI0005a9r were both detected for the first time in Paper I.

Based on our follow-up observations with Robo-AO, all four of these objects have no excess once the contribution from background objects is subtracted at W4. AWI0000uj2 is likely significantly contaminated at $W 4$ by two objects $\sim 4$ mag fainter than it in the Sloan $i$ band. AWI0004nfu exhibits seven background objects 3-9 mag fainter than it. AWI0005yiz exhibits several background objects $\sim 5-8$ mag fainter than it, and AWI0005a9r exhibits several background objects 3-10 mag fainter than it in Sloan $i$. Further analysis and observations (e.g., additional wavelength coverage of the background objects to confirm the shape of their SED) are required to confirm that the observed excess is significantly affected by these targets. However, these cases illustrate that highresolution follow-up can impact the quality of published infrared excesses.

\subsection{A Reassessment of a Previous WISE-based M-dwarf Disk Search}

M-dwarf disk systems are particularly invaluable systems to identify. M-dwarfs are key targets for large-scale exoplanet searches (e.g., Irwin et al. 2009; Ricker et al. 2014) and host some of the most unique exoplanetary systems discovered to date (e.g., Anglada-Escudé et al. 2016; Gillon et al. 2017). Debris disks around M-dwarfs should have the same informative powers as their higher-mass star cousins. The recent discovery of cold debris around the M-dwarf exoplanet host Proxima Centauri with the Atacama Large Millimeter Array (ALMA; Anglada et al. 2017) may yield valuable insights into the structure of that planetary system, though a recent reanalysis indicates that much of the flux previously attributed to dust can be attributed to a large flare (MacGregor et al. 2018).

However, the current sample of M-dwarf debris disk systems has significant shortcomings. Only $\sim 2 \%$ of $\mathrm{dM}$ stars are currently known to host debris disks (e.g., Deacon et al. 2013; Theissen \& West 2014; Boucher et al. 2016; Silverberg et al. 2016; Binks \& Jeffries 2017), less than the $\sim 14 \%$ expected from the mass distribution of primordial disks, and much less than the $20 \%$ fraction of A dwarfs with known disks (Greaves 2010). As part of our application of Disk Detective analysis to other surveys, we began a reanalysis of imagery of the M-dwarf disk candidates presented in Theissen \& West (2014), one of the largest lists of M-dwarf disk candidates from WISE published to date.

These disk candidates generally suffer from low $\mathrm{S} / \mathrm{N}$ at $W 4$. Of the 175 disk candidates presented in Theissen \& West (2014), only three meet the Disk Detective input catalog criterion of $\mathrm{w} 4 \mathrm{sn} r>10$. By contrast, 152 of the 175 only have upper limit magnitudes at $W 4$ due to $\mathrm{w} 4 \mathrm{sn} r<2$, as per the AllWISE data-release quality flags. In most cases, W4 postage-stamp images only show background emission, without a coherent point source at W4. This is presumably due in combination to the shallowness of the $W 4$ band and the distance of these targets.

Because of this lack of a point source, the Disk Detective classification method fails for the majority of these targets. Due to the shallowness of W4 compared to the other three WISE bands, Theissen \& West (2014) primarily focused on significant excess at $W 3$, noting that those targets with $\mathrm{w} 4 \mathrm{snr}>3$ and excess at $W 4$ also exhibited excess at $W 3$. However, the Disk Detective method is still viable for the 13 targets with $\mathrm{w} 4 \mathrm{snr}>3$.

We downloaded 1 arcmin postage-stamp images of the Theissen \& West (2014) disk candidates from the IRSA finder chart, using a blue-white color scale similar to that used on the Disk Detective website, and we applied a 12-arcsec-radius red circle to these images to effectively generate Disk Detective flip books of these targets. Our team of advanced users then analyzed these targets as if they appeared on the Disk Detective website and cataloged their assessment of these objects in the 
Table 4

Disk Candidates

\begin{tabular}{|c|c|c|c|c|c|c|c|c|c|}
\hline \multicolumn{3}{|c|}{ Identifiers } & \multirow{2}{*}{$\begin{array}{l}\text { Sp. } \\
\text { Type }^{\text {a }}\end{array}$} & \multirow{2}{*}{$\begin{array}{l}\text { Distance }^{\mathrm{b}} \\
\quad(\mathrm{pc})\end{array}$} & \multicolumn{4}{|c|}{ Magnitudes } & \multirow[b]{2}{*}{ Notes } \\
\hline Zooniverse & HD & WISEA & & & $\mathrm{Vmag}^{\mathrm{c}}$ & Jmag & W1mag & W4mag & \\
\hline AWI0000gib & 1777 & J002133.47-661816.6 & $\mathrm{A} 0 \mathrm{~V}$ & $163 \pm 10$ & $7.361 \pm 0.010$ & $7.365 \pm 0.027$ & $7.356 \pm 0.027$ & $6.793 \pm 0.066$ & \\
\hline AWI00062lo & 2830 & J003140.76-014737.3 & $\mathrm{A} 0 \mathrm{~V}$ & $109 \pm 6$ & $7.07 \pm 0.01$ & $6.917 \pm 0.019$ & $6.841 \pm 0.033$ & $5.832 \pm 0.049$ & $a, b$ \\
\hline AWI0005mry & 3051 & J003412.66+540359.0 & A1V & $213 \pm 15$ & $7.595 \pm 0.010$ & $7.350 \pm 0.019$ & $7.346 \pm 0.049$ & $6.881 \pm 0.067$ & a \\
\hline AWI0000jvv & & $\mathrm{J} 003507.14+070625.0$ & & & & $8.142 \pm 0.019$ & $6.937 \pm 0.033$ & $4.923 \pm 0.035$ & $\mathrm{c}$ \\
\hline AWI00055sx & 6370 & J010652.55+743754.5 & B9IV & $354 \pm 39$ & $8.368 \pm 0.012$ & $8.168 \pm 0.029$ & $8.149 \pm 0.023$ & $7.139 \pm 0.087$ & $\mathrm{a}$ \\
\hline
\end{tabular}

Notes. (a) Appears in Paper I. (b) Listed as a comoving object in Oh et al. (2017). (c) Listed in the K2 Ecliptic Plane Input Catalog. (d) Listed in Chen et al. (2016). (e) Identified in Vieira et al. (2003). (f) Identified in Corbally \& Straižys (2009). (g) Identified in Cotten \& Song (2016). (h) Identified in Esplin et al. (2014). (i) Identified in Rebull et al. (2011). (j) Identified in Wu et al. (2013). (k) Identified in Guieu et al. (2010). (1) Identified in Koenig et al. (2015). (m) Identified in Hernández et al. (2005). (n) Identified in Alcala et al. (1996). (o) Identified in Rojas et al. (2008). (p) Identified in Sanchez et al. (2014). (q) Identified in Rebull et al. (2000). (r) Identified in Megeath et al. (2012). (s) Identified in Luhman et al. (2008). (t) Identified in Rapson et al. (2014). (u) Identified in Rizzuto et al. (2012). (v) Identified in Chen et al. (2012). (w) Identified in Chen et al. (2014). (x) Identified in Melis et al. (2013). (y) Identified in Evans et al. (2009). (z) Identified in Evans et al. (2003). ( $\alpha$ ) Identified in Cruz-Saenz de Miera et al. (2014). ( $\beta$ ) Identified in Clarke et al. (2005). ( $\gamma$ ) Identified in Kennedy \& Wyatt (2013). ( $\delta$ ) Identified in Patel et al. (2014). ( $\epsilon$ ) Identified in Barentsen et al. (2011).

${ }^{a}$ Spectral types are from SIMBAD, with the exception of sources that appear in Paper I. For those sources, we use the spectral types published in that paper.

${ }^{\mathrm{b}}$ Distances are based on parallax measurements from Hipparcos or TGAS, as listed on SIMBAD.

${ }^{c}$ Sourced from SIMBAD.

(This table is available in its entirety in machine-readable form.)

Disk Detective website categories. All 13 objects with w4snr $>3$ were classified as extended by a majority of classifiers. Five of the 13 were also majority-classified as multiple, and two were also majority-classified as oval.

The disk candidates identified by the Theissen \& West (2014) M-dwarf survey typically do not meet the Disk Detective standard for inclusion for analysis; the 13 that meet these standards are clear false positives that do not survive Disk Detective's by-eye examination. It is possible that the remaining $162 \mathrm{M}$-dwarf disk candidates, with significant W3 excess and low $\mathrm{S} / \mathrm{N}$ at $W 4$, could represent a class of hot, distant debris disks for which Disk Detective is not designed. However, given the high false-positive rate of objects that can be assessed with the Disk Detective methodology, we recommend treating these results with caution.

\section{New Disk Candidates}

Based on our follow-up observations with Palomar/Robo$\mathrm{AO}$ and du Pont/Retrocam, we find that 244 of our observed targets, including 214 sources first identified by Disk Detective, have no significantly contaminating background objects within the $12^{\prime \prime}$ radius of the W4 PSF, giving us the confidence to publish them as disk candidates. The candidates are listed in Table 4; previous surveys that have also identified these targets are listed in the Notes column. We briefly summarize characteristics of some objects of particular interest in Appendix C.

We used available photometry from SIMBAD, 2MASS, and WISE (including corrected $W 1, W 2$, and $W 4$ photometry) to fit the SEDs of these systems. We initially fit the stellar component of the system with a blackbody to estimate the stellar temperature and ratio of stellar radius to distance. In the case where a blackbody fit yields a temperature less than $7000 \mathrm{~K}$, we instead fit with a stellar model from the BT-Settl CIFIST package (Baraffe et al. 2015), also fitting for $\log (g)$. We initially fit the three bluest photometry points, then refit including the next bluest point if it is not in significant excess, repeating the last step iteratively until the next point is either $W 3$ or in excess. We fit the remaining excess using a singletemperature blackbody to determine the dust temperature. We then find the best-fit parameters using the emcee package (Foreman-Mackey et al. 2013), using the previous parameter estimates as an initial guess. These SEDs, shown in Figure 6, provide a useful first-order estimate of these fundamental disk parameters. We list these parameters for likely debris disks in Table 5 and for likely YSO disks in Table 6. Table 5 consists of all candidates with only one point of excess (W4), as well as candidates with two points of excess and $L_{\text {ir }} / L_{\star}<10^{-3}$. Table 6 consists of all other objects. For objects with more than two points of excess, we also provide the spectral index $\alpha$ of a power-law fit to the object's WISE data as an estimate of YSO class.

Of the 244 targets presented here, 223 have parallax measurements from Hipparcos or the second data release from Gaia, listed in Table 4. The parallax measurements indicate that 30 of these systems lie within $125 \mathrm{pc}$, making them prime candidates for follow-up observation. We list these candidates in Table 7.

We can also use these parallaxes and 2MASS photometry to construct a Hertzsprung-Russell (HR) diagram for our disk candidates (shown in Figure 7). On this figure, point color corresponds to disk temperature, while point size corresponds to $L_{\text {ir }} / L_{\star}$. A gray curve shows the zero-age main sequence (Pecaut \& Mamajek 2013). The point in gray in the lower left indicates typical uncertainties, $\sim 0.04 \mathrm{mag}$ in $\left(J-K_{s}\right)$ color and $\sim 0.15 \mathrm{mag}$ in $M_{\mathrm{K}_{s}}$. Although some stars have $K$-band excesses that shift their points to the right in this diagram, Figure 7 reveals that none of the host stars with parallaxes has a main-sequence type later than roughly K7. Even with our "drain-the-lake" approach to searching for debris disks, the M-dwarf disks remain hidden, at least in this sample.

The diagram also reveals some potentially interesting outliers. The yellow point near the center of the figure is 

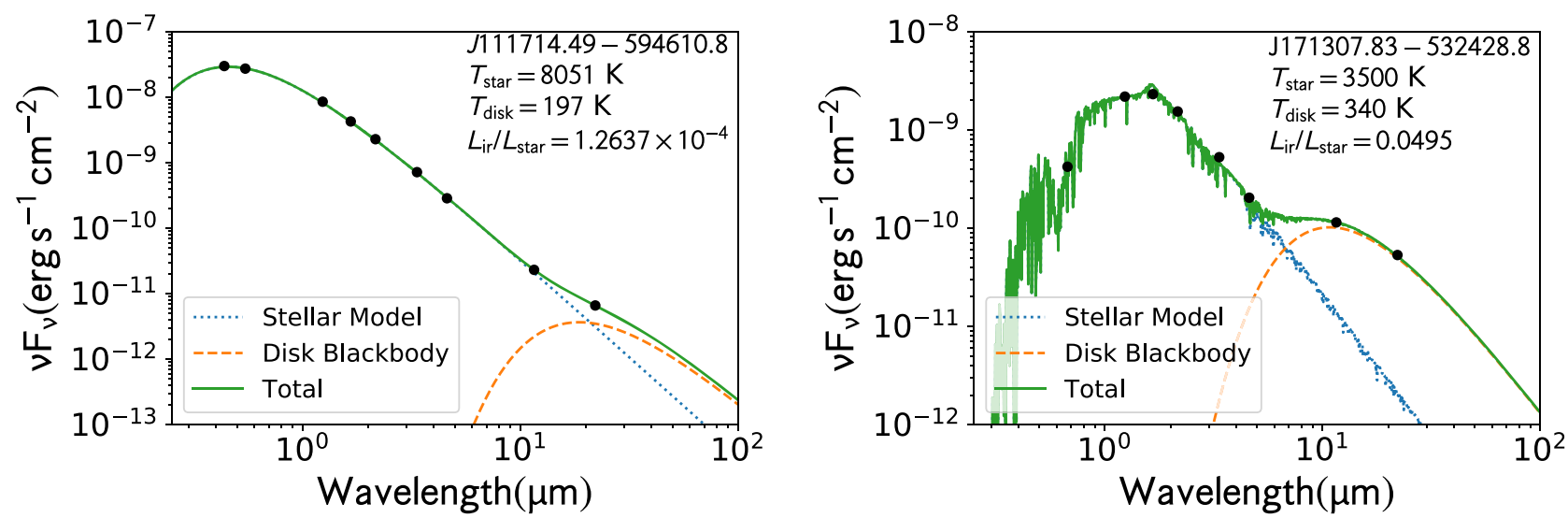

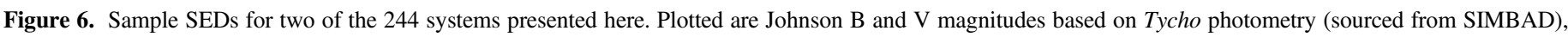

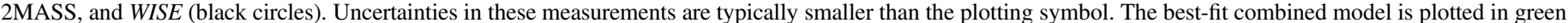

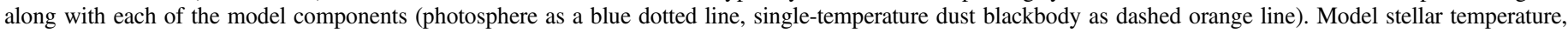

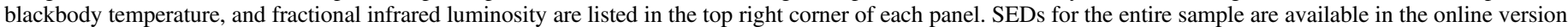
of the article.

(The complete figure set (245 images) is available.)

WISEA J191845.28+371449.2. This star, listed in SIMBAD as an A2 at a distance of $573 \mathrm{pc}$, has excess flux in all three 2MASS bands and all four WISE bands, which is well modeled by a single-temperature blackbody at $1535 \mathrm{~K}$ with $L_{\text {ir }} / L_{\star}=0.0647$ (Figure 8). The star lies roughly $0.7 \mathrm{mag}$ to the right of the zero-age main sequence primarily because of its $K_{s}$ excess of $0.623 \mathrm{mag}$. If this star is indeed a main-sequence star with a debris disk as its SED fit suggests, this system would be an example of an "extreme debris disk" (Meng et al. 2015; Theissen \& West 2017), that is, a signpost of a recent giant impact. Given the lack of an additional mid-IR-driven excess, this object could also be an example of a precursor to a two-component system with a hot debris disk (Akeson et al. 2009), where a gap has not yet been cleared between the two components. However, Gaia DR2 indicates that this object could be superluminous for a spectral type of $\sim \mathrm{A} 2$ (the expected spectral type for the SED-fit stellar temperature), and optical spectroscopy indicates that this object could instead be a weak-lined Herbig Ae star (A. S. Bans et al. 2018, in preparation). Additional follow-up with radio/submillimeter observations would be necessary to confirm whether this object hosts a warm debris disk, or instead has the expected substantial cold material reservoir of a Herbig Ae system.

Zuckerman (2015) noted the high frequency of warm dust disks occurring around stars that were members of binary systems. Oh et al. (2017) specifically searched for new comoving pairs and systems in the Tycho-Gaia Astrometric Solution (Gaia Collaboration et al. 2016a; Lindegren et al. 2016). Out of 619,618 stars searched, they identified 8472 stars as members of comoving pairs, and a further 2134 as members of larger comoving systems. We note that 27 of our disk candidates presented here appear as members of comoving systems in Oh et al. (2017; listed in Table 4), out of 105 with parallax data sufficient for inclusion in the Oh et al. (2017) survey. Twelve of these 105 , or $11 \% \pm 3 \%$, are members of comoving pairs only, significantly higher than the overall rate of $1.37 \%$ found by $\mathrm{Oh}$ et al. (2017). This significantly higher rate contributes further support to the hypothesis of Zuckerman (2015).

We tested the likelihood of moving group membership for each of the new disk candidates we present here using
BANYAN $\Sigma$ (Gagné et al. 2018), and we compared our targets with previous moving group membership determinations. We present the first $22 \mu \mathrm{m}$ excess detection around J111714.49-594610.8, a known member of the Lower Centaurus Crux (LCC). Testing its kinematics with BANYAN $\Sigma$, however, indicates a $44.9 \%$ probability of membership in LCC, and a $40.5 \%$ probability of membership in Carina. We also note the first detection of an infrared excess around J140353.79-534628.3, a known Sco-Cen member (Hoogerwerf 2000) for which BANYAN $\Sigma$ yields an $88.9 \%$ chance of membership in the Upper Centaurus-Lepus complex (UCL). We find that J164540.79-310226.6, a star with infrared excess previously detected by Cruz-Saenz de Miera et al. (2014), has a $96.9 \%$ probability of membership in the UCL. These determinations give us age benchmarks for these systems, allowing us to compare them to disk evolutionary models. Identification of disks in moving groups has yielded unexpected and valuable results with regard to theories of disk evolution, such as the identification of a primordial disk around an M-dwarf in the Carina association, at an age more than nine times greater than the $e$-folding time for primordial disk dissipation around solar-type stars (Silverberg et al. 2016; Murphy et al. 2018).

Most of our published targets do not yet have spectroscopically determined spectral types or luminosity classes, and many do not have measured distances. An ongoing spectroscopic follow-up campaign of DDOIs (A. S. Bans et al. 2018, in preparation) will present a more complete and more detailed analysis of the distribution of spectral types of our objects.

Between this paper, Paper I, and Silverberg et al. (2016), Disk Detective has now published 215 previously unidentified disk candidates. Of the 144 with either published spectral types or known parallaxes, the majority (110) are early-type main-sequence stars. A total of 125 of the 215 have disk temperatures $T_{\text {disk }}<300 \mathrm{~K}$, and disk temperatures range up to $1800 \mathrm{~K}$. A majority of these disks (114) have fractional infrared luminosities $L_{\mathrm{ir}} / L_{\mathrm{star}}>10^{-3}$, suggesting that these are likely primordial disks, per the criterion suggested by Williams \& Cieza (2011); The characteristics of this sample overall suggest that while designed to identify debris disks, Disk Detective also effectively locates new primordial disks. 
Table 5

Derived Parameters of Debris Disk Candidates

\begin{tabular}{lcccc}
\hline \hline Zooniverse & WISEA & $T_{\text {eff }}(\mathrm{K})$ & $T_{\text {disk }}(\mathrm{K})$ & \multicolumn{1}{c}{$L_{\text {ir }} / L_{\text {star }}$} \\
\hline AWI00062h7 & J002133.47-661816.6 & $11697_{-113}^{+112}$ & $216_{-24}^{+27}$ & $(2.8 \pm 0.4) \times 10^{-5}$ \\
AWI0000bs0 & J003140.76-014737.3 & $10319_{-69}^{+64}$ & $202 \pm 11$ & $(8.6 \pm 0.6) \times 10^{-5}$ \\
AWI0005mry & J003412.66+540359.0 & $9680 \pm 72$ & $>35$ & $<0.12$ \\
AWI0005yiv & J005926.26+400918.2 & $9165_{-52}^{+58}$ & $>46$ & $<0.005$ \\
AWI00055sx & J010652.55+743754.5 & $10106_{-85}^{+80}$ & $182_{-15}^{+16}$ & $(8.1 \pm 1.1) \times 10^{-5}$ \\
AWI00055sz & J011636.23+740136.6 & $9011_{-89}^{+99}$ & $685_{-113}^{+125}$ & $\left(4.5_{-1.3}^{+2.0} \times 10^{-4}\right.$ \\
\hline
\end{tabular}

(This table is available in its entirety in machine-readable form.)

\section{Summary}

In this paper, we presented the results of follow-up imaging of 261 Disk Detective Objects of Interest, determining whether background contaminants appeared and whether they significantly impacted the infrared excess around these objects observed with WISE. We find that background objects, while apparent in the images, significantly affect the observed excess at a rate of $\sim 6 \%$. Combining these data with false-positive rates from classifications and literature review, we find that AllWISE should yield $\sim 21,600$ high-quality disk candidates based on excess at $22 \mu \mathrm{m}$. Applying this result to other surveys, we estimate that $4 \%-8 \%$ of published disk candidates from highquality surveys may have background objects in follow-up high-resolution imaging bright enough to significantly affect the detected excess. Based on our expected yield of disks from AllWISE, we found that the searches of McDonald et al. (2012, 2017) and Marton et al. (2016) have lower-limit false-positive rates greater than $70 \%$. We considered the 175 disk candidates of Theissen \& West (2014) and found that the vast majority of these candidates would not be detected by Disk Detective due to insufficient $\mathrm{S} / \mathrm{N}$ at $W 4$. All 13 targets in the Theissen \& West (2014) search with W4 S/N sufficient for the Disk Detective methodology to apply were false-positive identifications after visual inspection.

We presented a sample of 244 disk candidates, vetted through visual inspection by citizen scientists and highresolution follow-up imaging to refine the observed excess. Disk Detective has now published 215 newly identified disk systems, of which 114 have fractional infrared luminosities indicative of primordial disks (either full protoplanetary or transitional). We find 12 of our disk candidates to be in comoving pairs, providing further support for the hypothesis of Zuckerman (2015) that there is a causal relationship between a distant companion and a warm, dusty debris disk. We identified one system, WISEA J191845.28+371449.2, as a likely "extreme" debris disk, based on its high fractional infrared luminosity. Thirty of these systems lie within $125 \mathrm{pc}$, including 26 debris disks. These nearby disk systems are good targets for adaptive optics and coronagraphic imaging to directly image exoplanets in orbit around the host star.

High-resolution follow-up imaging can eliminate many false positives, but it will not eliminate not-yet-identified spectroscopic false-positive detections of primordial and debris disks (e.g., previously unidentified dust-producing M giants, AGNs, classical Be stars). A spectroscopic follow-up program to identify these sorts of false positives is ongoing. The results presented here should be used in conjunction with the results of that program (A. S. Bans et al. 2018, in preparation) to determine the expected yield of AllWISE.
We thank the anonymous reviewer for providing comments that helped to improve the content and clarity of this paper, We acknowledge support from grant 14-ADAP14-0161 from the NASA Astrophysics Data Analysis Program and grant 16-XRP16_2-0127 from the NASA Exoplanets Research Program. M.J.K. acknowledges funding from the NASA Astrobiology Program via the Goddard Center for Astrobiology.

This publication makes use of data products from the Widefield Infrared Survey Explorer, which is a joint project of the University of California, Los Angeles, and the Jet Propulsion Laboratory (JPL)/California Institute of Technology (Caltech), and NEOWISE, which is a project of JPL/Caltech. WISE and NEOWISE are funded by NASA.

2MASS is a joint project of the University of Massachusetts and the Infrared Processing and Analysis Center (IPAC) at Caltech, funded by NASA and the NSF.

The Digitized Sky Survey was produced at the Space Telescope Science Institute under U.S. Government grant NAG $\mathrm{W}-2166$. The images of these surveys are based on photographic data obtained using the Oschin Schmidt Telescope on Palomar Mountain and the UK Schmidt Telescope. The plates were processed into the present compressed digital form with the permission of these institutions.

Funding for the SDSS and SDSS-II has been provided by the Alfred P. Sloan Foundation, the Participating Institutions, the National Science Foundation, the U.S. Department of Energy, the National Aeronautics and Space Administration, the Japanese Monbukagakusho, the Max Planck Society, and the Higher Education Funding Council for England. The SDSS website is http://www.sdss.org/.

The SDSS is managed by the Astrophysical Research Consortium for the Participating Institutions. The Participating Institutions are the American Museum of Natural History, Astrophysical Institute Potsdam, University of Basel, University of Cambridge, Case Western Reserve University, University of Chicago, Drexel University, Fermilab, the Institute for Advanced Study, the Japan Participation Group, Johns Hopkins University, the Joint Institute for Nuclear Astrophysics, the Kavli Institute for Particle Astrophysics and Cosmology, the Korean Scientist Group, the Chinese Academy of Sciences (LAMOST), Los Alamos National Laboratory, the Max-Planck-Institute for Astronomy (MPIA), the Max-PlanckInstitute for Astrophysics (MPA), New Mexico State University, Ohio State University, University of Pittsburgh, University of Portsmouth, Princeton University, the United States Naval Observatory, and the University of Washington.

This research has made use of the SIMBAD database, operated at CDS, Strasbourg, France. Some of the data presented in this paper were obtained from the Mikulski Archive for Space Telescopes (MAST). STScI is operated by 
Table 6

Derived Parameters of YSO Disk Candidates

\begin{tabular}{|c|c|c|c|c|c|}
\hline Zooniverse & WISEA & $T_{\text {eff }}(\mathrm{K})$ & $\alpha_{\text {disk }}$ & $T_{\text {disk }}(\mathrm{K})$ & $L_{\mathrm{ir}} / L_{\mathrm{star}}$ \\
\hline AWI0000nfp & J002155.14-672715.9 & $4700 \pm 100$ & $\ldots$ & $509 \pm 21$ & $(7.7 \pm 0.05) \times 10^{-3}$ \\
\hline AWI0000jvv & $\mathrm{J} 003507.14+070625.0$ & $3500 \pm 100$ & $\ldots$ & $368_{-11}^{+12}$ & $\left(1.23_{-0.04}^{+0.05}\right) \times 10^{-2}$ \\
\hline AWI00062m4 & J004826.42+020753.0 & $4600 \pm 100$ & $\cdots$ & $207_{-4}^{+5}$ & $\left(3.7_{-0.1}^{+0.1}\right) \times 10^{-3}$ \\
\hline AWI00062mq & J011743.47-523330.8 & $6400 \pm 100$ & $-0.12 \pm 0.01$ & $436 \pm 4$ & $(9.0 \pm 0.2) \times 10^{-2}$ \\
\hline AWI0005aeg & J013833.77+780834.3 & $6700 \pm 100$ & $-2.11 \pm 0.02$ & $1210_{-25}^{+26}$ & $\left(2.5_{-0.1}^{+0.2}\right) \times 10^{-2}$ \\
\hline
\end{tabular}

(This table is available in its entirety in machine-readable form.)

the Association of Universities for Research in Astronomy, Inc., under NASA contract NAS5-26555. Support for MAST for non-HST data is provided by the NASA Office of Space Science via grant NNX13AC07G and by other grants and offices. This research has made use of the VizieR catalog access tool, CDS, Strasbourg, France.

This work has made use of data from the European Space Agency (ESA) mission Gaia (https://www.cosmos.esa.int/ gaia), processed by the Gaia Data Processing and Analysis Consortium (DPAC; https://www.cosmos.esa.int/web/gaia/ dpac/consortium). Funding for the DPAC has been provided by national institutions, in particular the institutions participating in the Gaia Multilateral Agreement.

The Robo-AO system was developed by collaborating partner institutions, the California Institute of Technology, and the Inter-University Centre for Astronomy and Astrophysics, and with the support of the National Science Foundation under grants AST-0906060, AST-0960343 and AST-1207891, the Mt. Cuba Astronomical Foundation, and by a gift from Samuel Oschin. C.B. acknowledges support from the Alfred P. Sloan Foundation.

IRAF is distributed by the National Optical Astronomy Observatory, which is operated by the Association of Universities for Research in Astronomy (AURA) under a cooperative agreement with the National Science Foundation. PyRAF is a product of the Space Telescope Science Institute, which is operated by AURA for NASA. This research made use of ds9, a tool for data visualization supported by the Chandra X-ray Science Center (CXC) and the High Energy Astrophysics Science Archive Center (HEASARC) with support from the JWST Mission office at the Space Telescope Science Institute for 3D visualization. Resources supporting this work were provided by the NASA High-End Computing (HEC) Program through the NASA Center for Climate Simulation (NCCS) at Goddard Space Flight Center.

Facilities: FLWO:2MASS, CTIO:2MASS, WISE, PO:1.5 m (Robo-AO), du Pont (RetroCam).

Software: IRAF (Tody 1993), PyRAF, AstroPy (Astropy Collaboration et al. 2013), NumPy (Van Der Walt et al. 2011), SciPy (Jones et al. 2001), Matplotlib (Hunter 2007), pandas (McKinney 2010), emcee (Foreman-Mackey et al. 2013).

\section{Appendix A}

\section{Changes to the Website Classification Scheme after Paper I}

Since Paper I, we have made two key changes to the website classification setup: we changed the scheme for retiring subjects, and we corrected the online photometry to account for saturation effects at $W 1$ and $W 2$.

\section{A.1. New Retirement Scheme}

Visitors to the diskdetective.org site ("users") view "flip books" showing several images of the same source at different wavelengths. After they view the flip books, users answer a question, "What best describes the object you see?," by clicking on one or more of six buttons, labeled "Multiple Objects in the Red Circle," "Object Moves off the Crosshairs," "Extended beyond Circle in WISE Images," "Empty Circle in WISE Images," "Not Round in DSS2 or 2MASS Images," and "None of the Above/Good Candidate." With the exception of the "None of the Above" option, the user can choose more than one description per flip book. After at least one of these classification buttons is chosen, a button labeled "Finish" becomes active; clicking this button records the user's choices and causes the next flip book to appear.

Utilizing this classification data requires a "retirement scheme," a set of rules for deciding when a given subject has received enough classifications that we can be reasonably sure that it is a good candidate. Prior to 2015 July 23, we used a very simple retirement scheme: we retired all subjects after 15 classifications. However, we realized that certain kinds of sources did not require so many independent classifications to make a confident decision about their nature. So on 2015 July 23 , we put in place a new retirement scheme that allows us to progress more rapidly through the data.

To develop this new scheme, we experimented with several possible retirement rules by applying them to a set of subjects that already had 15 classifications, to see if they would alter the final classification. We found that users were especially reliable at classifications as "Multiple Objects in the Red Circle," or "Not Round in DSS2 or 2MASS images." Even if we used only the first five classifications of subjects in these categories, it did not change their ultimate classification. So we chose to implement a retirement rule that retires the subjects as either "multiple objects..." or "not round..." when four out of the first five classifications are either "multi" or "oval," respectively. From the first year of classifications, we know that almost $45 \%$ of our sources fit in one of these two categories. So we expected a substantial increase in efficiency from this rule, and indeed, though the number of active participants in the project has remained stable roughly since July 2014 , we have seen a noticeable increase in the retirement rate since implementing the new rule, so $26 \%$ of our 278,121 subjects have now been retired.

\section{A.2. Removal of WISE 1 Dropouts}

A second improvement we made to the website was that we removed a list of problematic subjects from the online classification process. As Patel et al. (2014) and others have 
Table 7

Disk Candidates within $125 \mathrm{pc}$

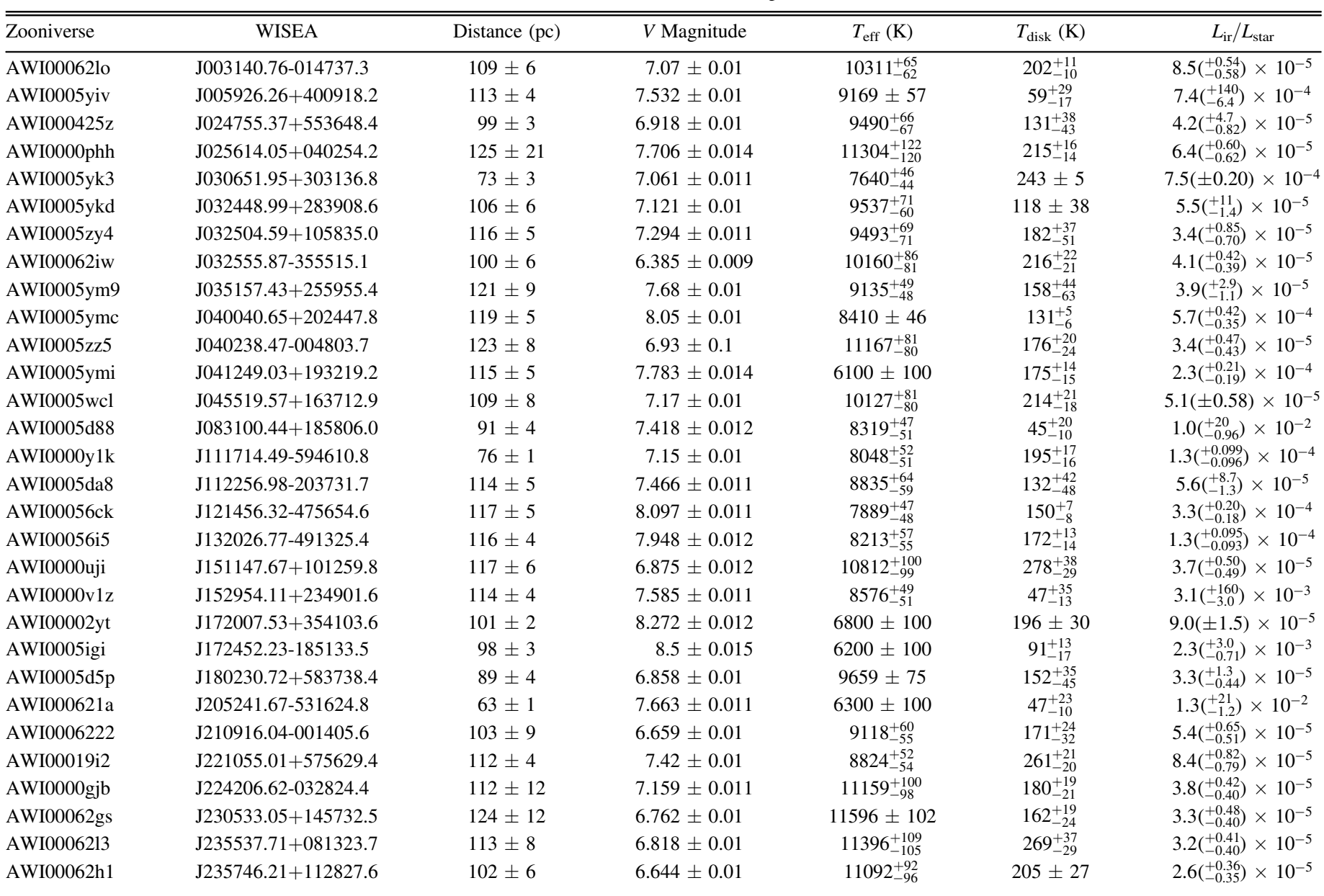

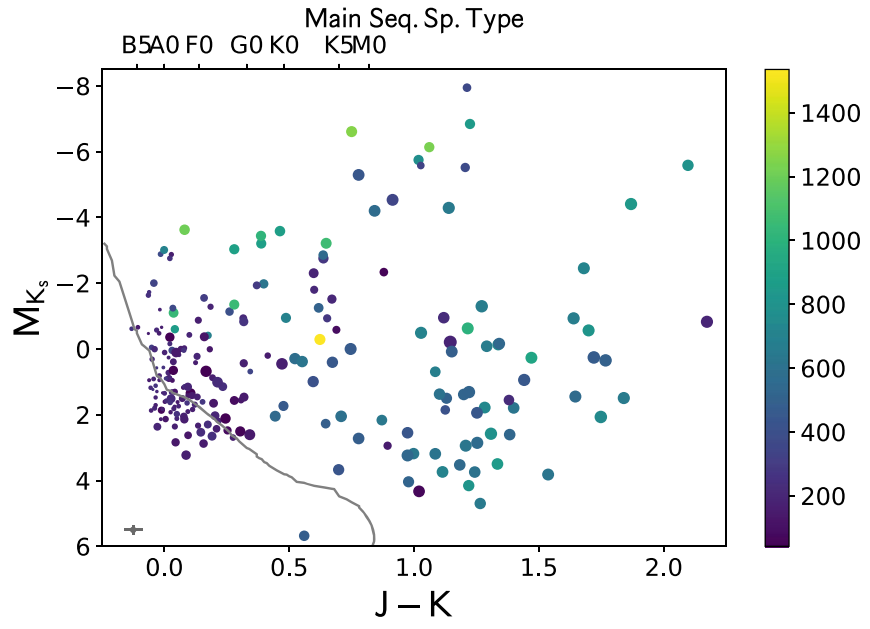

Figure 7. $2 \mathrm{MASS}\left(M_{K}, J-K\right)$ color-magnitude diagram of 223 disk candidates with parallax measurements. Point color corresponds to disk temperature, while point size corresponds to $L_{\text {ir }} / L_{\text {star. }}$. We find that 148 disk candidates lie within $1.5 \mathrm{mag}$ of the zero-age main sequence (gray line), and 126 lie within $1.5 \mathrm{mag}$ of the zero-age main sequence with spectral type earlier than G0. Main-sequence spectral types are listed across the top for reference. The point in gray in the lower left indicates typical uncertainties, $\sim 0.04 \mathrm{mag}$ in $(J-K)$ color and $\sim 0.15 \mathrm{mag}$ in $M_{K}$.

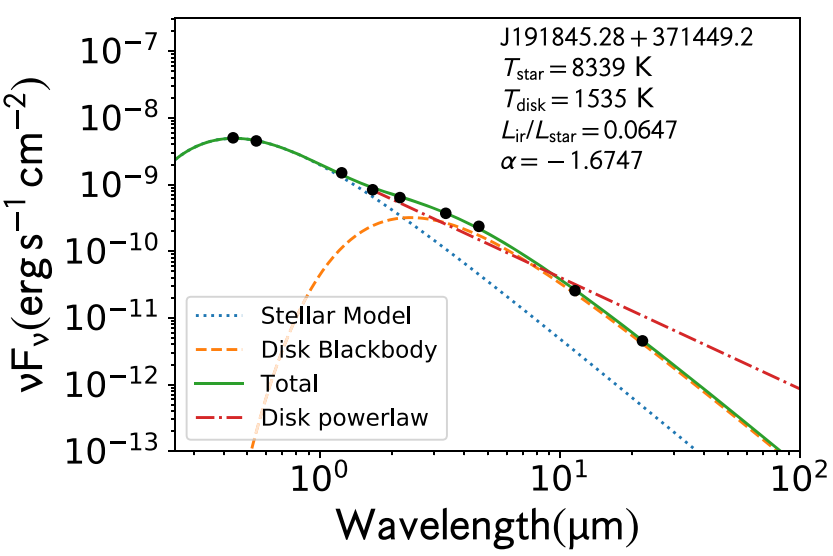

Figure 8. SED for WISEA J191845.28+371449.2. This system is well fit by a stellar temperature blackbody with a $\sim 1535 \mathrm{~K}$ disk blackbody with fractional infrared luminosity $\sim 0.065$, suggesting an extreme debris disk.

noted, bright sources can saturate the WISE detectors, causing systematic errors in the WISE photometry. These errors are worse at the WISE $W 1$ and $W 2$ bands. Since we chose objects for our input catalog using [W1]-[W4] colors, these 
photometric errors caused us to include some objects in our input catalog that had no true excess at $W 4(22 \mu \mathrm{m})$, only false deficits at $W 1$. Using the saturation corrections in Patel et al. (2014), we found a list of 279 subjects that had been included in our search incorrectly because of saturation errors in their $W 1$ photometry. On 2015 August 3, we removed these sources from the vetting process.

\section{Appendix B}

\section{Deriving Brightness Thresholds for Contaminants}

In this section, we derive a minimum brightness for background objects to produce a false-positive excess detection in our system. We also show how these brightnesses propagate to the $i, Y$, and $H$ bands we use in our follow-up observations.

\section{B.1. Minimum Contaminant Brightness in W4}

Let us suppose that we have a target star with no circumstellar dust, whose spectrum is accurately approximated by the Rayleigh-Jeans law in the WISE bands. We will denote this target star's intrinsic magnitudes as $m_{t}$ and its intrinsic flux as $f_{m, t}$. Let us also suppose a background contaminant whose intrinsic magnitudes will be denoted $m_{c}$ and whose flux will be denoted $f_{m, c}$. This background contaminant lies substantially inside the W4 PSF HWHM, such that the observed W4 magnitude $[W 4]_{\text {obs }}$ is the magnitude of the combined light from the target and contaminant,

$$
f_{W 4, \mathrm{obs}}=f_{W 4, t}+f_{W 4, c},
$$

but outside the $W 1$ PSF HWHM, such that $[W 1]_{\mathrm{obs}}=[W 1]_{t}$.

Assuming that our target star has $\left[W 1_{t}\right]-\left[W 4_{t}\right]=0$, we wish to know the minimum brightness $W 4_{c}$ such that $\left[W 1_{\text {obs }}\right]-\left[W 4_{\text {obs }}\right] \geqslant 0.25$.

Making substitutions suggested by the above equalities, we have

$$
W 4_{t}-W 4_{\text {obs }} \geqslant 0.25 \text {. }
$$

Using the equation for flux/magnitude conversion, this becomes

$$
f_{W 4, c} \geqslant 0.258925 f_{W 4, t} .
$$

Converting this back into magnitude differences, we have

$$
W 4_{c}-W 4_{t} \leqslant 1.467 .
$$

We thus show that contaminants more than $\sim 1.5$ mag dimmer than the target star in W4 will not produce a significant enough excess for the star to become a false-positive entry in the Disk Detective Input Catalog. As such, with the greater depth probed by high-resolution imaging, we must quantitatively assess whether a detected background contaminant will produce a false positive, rather than qualitatively assessing it, as was done with the website-based classifications (Binks 2016).

\section{B.2. Applying the Minimum Brightness to the $\mathrm{i}, \mathrm{Y}$, and $\mathrm{H}$ Bands}

To determine if a potential background object is bright enough to produce a false-positive excess in $W 4$, we must know the difference in magnitude between such a contaminant and the target in the bands in which we conducted highresolution follow-up observations. We assume for this exercise that the target's optical and near-IR SED is approximately identical to an idealized Vega; that is, it is of zeroth magnitude in all bands. This sets the contaminating magnitude limit of 1.467. Below, we give examples of three typical contaminants,
Table 8

W4 Colors of Selected Background Object Types

\begin{tabular}{lccc}
\hline \hline & \multicolumn{3}{c}{ Colors } \\
\cline { 2 - 4 } Characteristics & $i-W 4$ & $Y_{C}-W 4$ & $H_{C}-W 4$ \\
\hline M-dwarf: $T_{\text {eff }}=3300 \mathrm{~K}, \log (g)=5.0$ & 3.545 & 1.896 & 0.801 \\
Class I YSO: $\lambda f_{\lambda} \sim \lambda^{0}$ & 2.94 & 2.05 & 1.82 \\
ULIRG: $\lambda f_{\lambda} \sim \lambda^{2}$ & 10.25 & 8.67 & 7.48
\end{tabular}

each with a $W 4$ magnitude of 1.467: a background M-dwarf and two different power-law spectra. Table 8 lists the $i, Y$, and $H$ magnitudes for these objects.

\section{B.2.1. False Positive That Is Due to a Background M-dwarf}

The initial mass function of the neighborhood peaks at a spectral type of M2-M3.5, which corresponds to a stellar effective temperature of $T_{\text {eff }} \simeq 3250-3400 \mathrm{~K}$. Accordingly, we select a model M-dwarf atmosphere with $T_{\text {eff }}=3300 \mathrm{~K}$ and $\log (g)=5.0$ from the BT-Settl package of model atmospheres (Baraffe et al. 2015) as our contaminating M-dwarf. The BTSettl models have precomputed magnitudes for many filter systems, which we use here to compute colors. This model has a $[H]-[W 4]$ value of 0.801 , corresponding to an $H$-band contaminant delta magnitude of 2.268 mag. The [i]-[W4] value for this model is $3.545 \mathrm{mag}$, yielding a magnitude difference in Robo-AO data of 5.012 mag.

\section{B.2.2. False Positive That Is Due to Background Sources with Power- law Spectra}

Some objects are reasonably represented with a power-law spectrum $\lambda f_{\lambda} \sim \lambda^{\alpha}$. For these objects, colors can to first order be approximated as

$$
\left[m_{\lambda_{1}}\right]-\left[m_{\lambda_{2}}\right] \sim 2.5 \log _{10}\left(\frac{f_{0, \lambda_{1}}}{f_{0, \lambda_{2}}}\right)-2.5(\alpha-1) \log _{10} \frac{\lambda_{1}}{\lambda_{2}} .
$$

For the colors of interest in this paper, this corresponds to

$$
\begin{aligned}
& {[H]-[W 4] \sim 1.817+2.831 \alpha,} \\
& {[Y]-[W 4] \sim 2.048+3.313 \alpha,}
\end{aligned}
$$

and

$$
[i]-[W 4] \sim 2.940+3.654 \alpha .
$$

Young stellar objects are defined by the value of $\alpha$; a Class I YSO has slope $\alpha=0$ (Lada 1987; Kenyon \& Hartmann 1995). This spectrum also roughly approximates a heavily reddened early-type star. If such an object were in the background of our images and bright enough to produce a false positive, the delta magnitude in the $H$ band would be 3.284, and the $Y$-band delta magnitude would be 3.515 . These could both likely be detected in our images. The $i$-band delta magnitude would be 4.407 , also likely detectable.

Also of interest is the case where $\alpha=2$. This roughly corresponds to the SED of a (U)LIRG, which has an AGN component (Vega et al. 2008). In this case, however, the $H$-band delta magnitude would be 8.946 and the $Y$-band delta magnitude would be 10.141 , while the $i$-band delta magnitude would be 11.715. These are all clearly undetectable in our follow-up image data and are thus treated instead with the prescription of Papovich et al. (2004) in Section 5.2. 


\section{Appendix C Comments on Selected Disk Candidates}

Below are brief comments on selected disk candidates presented in Section 7. Having noted previous identifications as disk candidates in Section 7, we primarily discuss characteristics of the subject's appearance, either in the images used on the Disk Detective website or in the follow-up images.

1. J021327.01+421923.3. This system, which was previously identified by Cotten \& Song (2016) and McDonald et al. (2017), exhibits slight extension in the W4 image.

2. J023720.84+395345.8. This system is a known spectroscopic binary (Hube 1981). The companion, an early-G main-sequence star based on the binary mass function, has a projected angular separation of $\ll 1^{\prime \prime}$, making it undetectable as a separate component in follow-up data. The SED indicates no significant effect on the $22 \mu \mathrm{m}$ excess.

3. J025926.83+593531.6. We recover this system, a spectroscopic binary (Abt 2009), that was previously identified as a source in the $W 5$ region by Koenig et al. (2008). The secondary component has a minimum mass of $1.7326 R_{\odot}$ based on the binary mass function, suggesting a possible A7V star. The projected angular separation is $\ll 1^{\prime \prime}$, making it undetectable as a separate component in follow-up data. The SED indicates no significant effect on the $22 \mu \mathrm{m}$ excess.

4. J030854.20-185809.1. This system, a known A0V system, is best fit as a two-stellar-component system with a K dwarf component, as well as a $349 \mathrm{~K}$ disk. Further observation is necessary to confirm this additional component.

5. J034400.28+243324.6. This $\delta$ Scuti variable, which appears in Cotten \& Song (2016) and McDonald et al. (2017), exhibits slight extension at $W 4$.

6. J041517.47+505124.0. This system, which appears in Marton et al. (2016), has been identified as a Be star previously (Merrill \& Burwell 1943) and more recently as having $\mathrm{H} \alpha$ in emission (Kohoutek \& Wehmeyer 1999). Further spectroscopic follow-up is necessary to determine the nature of this emission (i.e., whether the target is a classical Be star, rather than a debris disk host).

7. J043521.12-081730.0. This object exhibits slight extension at $W 4$.

8. J051143.75+122012.5. This system exhibits slight extension at $W 4$.

9. J051328.63-043910.6. This object is a component of a binary system. Its companion is $35^{\prime \prime}$ away from the source, too distant to affect the observed excess.

10. J052331.01-010423.6. This star exhibits eclipses from circumstellar material, per Osborn et al. (2017).

11. J053707.15+603636.4. This object, which appears in Cotten \& Song (2016), exhibits slight extension at W4.

12. J054330.38+251724.4. This system has previously been identified as an $\mathrm{H} \alpha$ emitter (Kohoutek \& Wehmeyer 1999). Further spectroscopic follow-up is necessary to determine the nature of this emission.

13. J054733.26+521144.5. This system has previously been identified as an $\mathrm{H} \alpha$ emitter (Merrill \& Burwell 1950). Further spectroscopic follow-up is necessary to determine the nature of this emission.
14. J111714.49-594610.8. This target is a member of the LCC of the Sco-Cen OB2 association, per Hoogerwerf (2000). Testing its kinematics with BANYAN $\Sigma$ yields a $44.9 \%$ membership probability in LCC and a $40.5 \%$ membership probability in Carina.

15. J111925.92-301922.9 and J165204.85+145827.2. We report the first detection of a disk around the $\alpha^{2} \mathrm{CVn}$ variable AWI0005cwg. We also note that AWI00004o8, identified in Paper I, is also an $\alpha^{2} \mathrm{CVn}$ variable.

16. J114336.83-802900.5. This target exhibits slight extension at $W 4$.

17. J132026.77-491325.4. This target is a known diskhosting member of Sco-Cen (Chen et al. 2012). Reevaluation with BANYAN $\Sigma$ yields an $88.7 \%$ probability of membership in LCC.

18. J134909.18-541342.3. This target is a known diskhosting member of Sco-Cen (Chen et al. 2012). Reevaluation with BANYAN $\Sigma$ yields a $42.1 \%$ membership probability in LCC and a $49.5 \%$ membership probability in UCL.

19. J144458.63-280251.9. This target is a known multiple system (Horch et al. 2011) with an excess first detected by Cruz-Saenz de Miera et al. (2014). We do not resolve both components of the binary system.

20. J173254.69+404312.3. This target exhibits very slight extension at $W 4$.

21. J173832.90+425112.9. This target is a known Cepheid variable.

22. J183311.41+025439.0. This object shows a faint background object in DSS2 survey data that does not appear in Robo-AO observations.

23. J185211.39+102422.6. This object shows a faint background object in DSS2 survey data that does not appear in Robo-AO observations.

24. J190901.24+110641.3. This target exhibits $\mathrm{H} \alpha$ in emission (Kohoutek \& Wehmeyer 1999).

25. J192136.46+220744.7. This object shows a faint background object in DSS2 survey data that does not appear in Robo-AO observations.

26. J192437.52+563454.9. This object shows a very faint background object in DSS2 survey data, which does not appear in the Robo-AO data for the system.

27. $J 210144.07+521717.6$. This object is an emission-line star (Kohoutek \& Wehmeyer 1999).

28. J212952.96+525601.9. This object exhibits a slight asymmetrical extension at $W 4$.

29. J215947.70-593411.9. This target is a known $\delta$ Scuti variable (Rodríguez et al. 2000).

30. J230112.67-585821.9. We note slight extension at W4.

\section{ORCID iDs}

Steven M. Silverberg (iD https://orcid.org/0000-0002-3741-4181 Marc J. Kuchner (iD https://orcid.org/0000-0002-2387-5489 John P. Wisniewski (i) https://orcid.org/0000-0001-9209-1808 Alissa S. Bans (iD https://orcid.org/0000-0003-0426-7987 John H. Debes (i) https://orcid.org/0000-0002-1783-8817 Scott J. Kenyon (iD https://orcid.org/0000-0003-0214-609X Christoph Baranec (i) https://orcid.org/0000-0002-1917-9157 Reed Riddle (1) https://orcid.org/0000-0002-0387-370X Nicholas Law (i) https://orcid.org/0000-0001-9380-6457 Milton K. D. Bosch (1) https://orcid.org/0000-0002-9766-2400 
Katharina Doll (ㄴ) https://orcid.org/0000-0002-2993-9869 Hugo A. Durantini Luca $\mathbb{1}$ https://orcid.org/0000-00024143-2550

Michiharu Hyogo (ํ) https://orcid.org/0000-0001-8343-0820

\section{References}

Abt, H. A. 2009, ApJS, 180, 117

Akeson, R. L., Ciardi, D. R., Millan-Gabet, R., et al. 2009, ApJ, 691, 1896 Alcala, J. M., Terranegra, L., Wichmann, R., et al. 1996, A\&AS, 119, 7 Anglada-Escudé, G., Amado, P. J., Barnes, J., et al. 2016, Natur, 536, 437 Anglada, G., Amado, P. J., Ortiz, J. L., et al. 2017, ApJL, 850, L6 Astropy Collaboration, Robitaille, T. P., Tollerud, E. J., et al. 2013, A\&A, 558, A 33

Avenhaus, H., Schmid, H. M., \& Meyer, M. R. 2012, A\&A, 548, A105 Baraffe, I., Homeier, D., Allard, F., \& Chabrier, G. 2015, A\&A, 577, A42 Baranec, C., Riddle, R., Law, N. M., et al. 2014, ApJ, 790, L8

Barentsen, G., Vink, J. S., Drew, J. E., et al. 2011, MNRAS, 415, 103

Binks, A. 2016, in IAU Symp. 314, Young Stars \& Planets Near the Sun, ed. J. H. Kastner, B. Stelzer, \& S. A. Metchev (Cambridge: Cambridge Univ. Press), 159

Binks, A. S., \& Jeffries, R. D. 2017, MNRAS, 469, 579

Borucki, W. J., Koch, D., Basri, G., et al. 2010, Sci, 327, 977

Boucher, A., Lafrenière, D., Gagné, J., et al. 2016, ApJ, 832, 50

Chen, C. H., Mittal, T., Kuchner, M., et al. 2014, ApJS, 211, 25

Chen, C. H., Pecaut, M., Mamajek, E. E., Su, K. Y. L., \& Bitner, M. 2012, ApJ, 756, 133

Chen, P. S., Shan, H. G., \& Zhang, P. 2016, NewA, 44,

Clarke, A. J., Oudmaijer, R. D., \& Lumsden, S. L. 2005, MNRAS, 363, 1111 Corbally, C. J., \& Straižys, V. 2009, BaltA, 18, 1

Cotten, T. H., \& Song, I. 2016, ApJS, 225, 15

Cruz-Saenz de Miera, F., Chavez, M., Bertone, E., \& Vega, O. 2014, MNRAS, 437, 391

Deacon, N. R., Schlieder, J. E., Olofsson, J., Johnston, K. G., \& Henning, T. 2013, MNRAS, 434, 1117

Debes, J. H., Hoard, D. W., Kilic, M., et al. 2011, ApJ, 729, 4

Esplin, T. L., Luhman, K. L., \& Mamajek, E. E. 2014, ApJ, 784, 126

Evans, N. J., II, Allen, L. E., Blake, G. A., et al. 2003, PASP, 115, 965

Evans, N. J., II, Dunham, M. M., Jørgensen, J. K., et al. 2009, ApJS, 181, 321

Foreman-Mackey, D., Conley, A., Meierjurgen Farr, W., et al. 2013, emcee: The MCMC Hammer, Astrophysics Source Code Library, ascl:1303.002

Gagné, J., Mamajek, E. E., Malo, L., et al. 2018, ApJ, 856, 23

Gaia Collaboration, Brown, A. G. A., Vallenari, A., et al. 2016a, A\&A, 595, A2 Gaia Collaboration, Prusti, T., de Bruijne, J. H. J., et al. 2016b, A\&A, 595, A1 Gillon, M., Triaud, A. H. M. J., Demory, B.-O., et al. 2017, Natur, 542, 456 Greaves, J. S. 2010, MNRAS, 409, L44

Guieu, S., Rebull, L. M., Stauffer, J. R., et al. 2010, ApJ, 720, 46

Hernández, J., Calvet, N., Hartmann, L., et al. 2005, AJ, 129, 856

Høg, E., Fabricius, C., Makarov, V. V., et al. 2000, A\&A, 355, L27

Hoogerwerf, R. 2000, MNRAS, 313, 43

Horch, E. P., Gomez, S. C., Sherry, W. H., et al. 2011, AJ, 141, 45

Hube, D. P. 1981, A\&AS, 44, 59

Hunter, J. D. 2007, CSE, 9, 90

Irwin, J., Charbonneau, D., Nutzman, P., \& Falco, E. 2009, in IAU Symp. 253, Transiting Planets, ed. F. Pont, D. Sasselov, \& M. J. Holman (Cambridge: Cambridge Univ. Press), 37

Jones, E., Oliphant, T., Peterson, P., et al. 2001, SciPy: Open Source Scientific Tools for Python, http://www.scipy.org

Kennedy, G. M., \& Wyatt, M. C. 2012, MNRAS, 426, 91

Kennedy, G. M., \& Wyatt, M. C. 2013, MNRAS, 433, 2334

Kenyon, S. J., \& Hartmann, L. 1995, ApJS, 101, 117

Koenig, X., Hillenbrand, L. A., Padgett, D. L., \& DeFelippis, D. 2015, AJ, 150,100

Koenig, X. P., Allen, L. E., Gutermuth, R. A., et al. 2008, ApJ, 688, 1142 Koenig, X. P., \& Leisawitz, D. T. 2014, ApJ, 791, 131

Koenig, X. P., Leisawitz, D. T., Benford, D. J., et al. 2012, ApJ, 744, 130

Kohoutek, L., \& Wehmeyer, R. 1999, A\&AS, 134, 255
Kuchner, M. J., Silverberg, S. M., Bans, A. S., et al. 2016, ApJ, 830, 84 Lada, C. J. 1987, in IAU Symp. 115, Star-forming Regions, ed. M. Peimbert \& J. Jugaku (Dordrecht: Reidel), 1

Lestrade, J.-F., Wyatt, M. C., Bertoldi, F., Menten, K. M., \& Labaigt, G. 2009, A\&A, 506, 1455

Lindegren, L., Lammers, U., Bastian, U., et al. 2016, A\&A, 595, A4

Liu, W. M., Padgett, D. L., Leisawitz, D., Fajardo-Acosta, S., \& Koenig, X. P. 2011, ApJL, 733, L2

Liu, W. M., Padgett, D. L., Terebey, S., et al. 2014, AJ, 147, 133

Luhman, K. L., Hernández, J., Downes, J. J., Hartmann, L., \& Briceño, C. 2008, ApJ, 688, 362

MacGregor, M. A., Weinberger, A. J., Wilner, D. J., Kowalski, A. F., \& Cranmer, S. R. 2018, ApJL, 855, L2

Marton, G., Tóth, L. V., Paladini, R., et al. 2016, MNRAS, 458, 3479

McDonald, I., Zijlstra, A. A., \& Boyer, M. L. 2012, MNRAS, 427, 343

McDonald, I., Zijlstra, A. A., \& Watson, R. A. 2017, MNRAS, 471, 770

McKinney, W. 2010, in Proc. IX Python in Science Conf., ed. S. van der Walt \&

J. Millman (Greenwich, CT: AQR Capital Management, LLC), 51, http:// conference.scipy.org/proceedings/scipy2010/mckinney.html

Megeath, S. T., Gutermuth, R., Muzerolle, J., et al. 2012, AJ, 144, 192

Melis, C., Zuckerman, B., Rhee, J. H., et al. 2013, ApJ, 778, 12

Meng, H. Y. A., Su, K. Y. L., Rieke, G. H., et al. 2015, ApJ, 805, 77

Merrill, P. W., \& Burwell, C. G. 1943, ApJ, 98, 153

Merrill, P. W., \& Burwell, C. G. 1950, ApJ, 112, 72

Morey, É., \& Lestrade, J.-F. 2014, A\&A, 565, A58

Murphy, S. J., Mamajek, E. E., \& Bell, C. P. M. 2018, MNRAS, 476, 3290

Oh, S., Price-Whelan, A. M., Hogg, D. W., Morton, T. D., \& Spergel, D. N. 2017, AJ, 153, 257

Osborn, H. P., Rodriguez, J. E., Kenworthy, M. A., et al. 2017, MNRAS, 471,740

Papovich, C., Dole, H., Egami, E., et al. 2004, ApJS, 154, 70

Patel, R. I., Metchev, S. A., \& Heinze, A. 2014, ApJS, 212, 10

Patel, R. I., Metchev, S. A., \& Heinze, A. 2015, ApJS, 220, 21

Patel, R. I., Metchev, S. A., Heinze, A., \& Trollo, J. 2017, AJ, 153, 54

Pecaut, M. J., \& Mamajek, E. E. 2013, ApJS, 208, 9

Plavchan, P., Jura, M., \& Lipscy, S. J. 2005, ApJ, 631, 1161

Plavchan, P., Werner, M. W., Chen, C. H., et al. 2009, ApJ, 698, 1068

Rapson, V. A., Pipher, J. L., Gutermuth, R. A., et al. 2014, ApJ, 794, 124

Rebull, L. M., Hillenbrand, L. A., Strom, S. E., et al. 2000, AJ, 119, 3026

Rebull, L. M., Koenig, X. P., Padgett, D. L., et al. 2011, ApJS, 196, 4

Rheault, J.-P., Mondrik, N. P., DePoy, D. L., Marshall, J. L., \& Suntzeff, N. B. 2014, Proc. SPIE, 9147, 91475L

Ricker, G. R., Winn, J. N., Vanderspek, R., et al. 2014, Proc. SPIE, 9143, 914320

Rizzuto, A. C., Ireland, M. J., \& Zucker, D. B. 2012, MNRAS, 421, L97

Rodríguez, E., López-González, M. J., \& López de Coca, P. 2000, A\&AS, 144,469

Rojas, G., Gregorio-Hetem, J., \& Hetem, A. 2008, MNRAS, 387, 1335

Sanchez, N., Inés Gómez de Castro, A., Lopez-Martinez, F., \& López-Santiago, J. 2014, A\&A, 572, A89

Silverberg, S. M., Kuchner, M. J., Wisniewski, J. P., et al. 2016, ApJL, 830, L28

Theissen, C. A., \& West, A. A. 2014, ApJ, 794, 146

Theissen, C. A., \& West, A. A. 2017, AJ, 153, 165

Tody, D. 1993, in ASP Conf. Ser. 52, Astronomical Data Analysis Software and Systems II, ed. R. J. Hanisch, R. J. V. Brissenden, \& J. Barnes (San Francisco, CA: ASP), 173

Van Der Walt, S., Colbert, S. C., \& Varoquaux, G. 2011, CSE, 13, 22

van Leeuwen, F. 2007, A\&A, 474, 653

Vega, O., Clemens, M. S., Bressan, A., et al. 2008, A\&A, 484, 631

Vieira, S. L. A., Corradi, W. J. B., Alencar, S. H. P., et al. 2003, AJ, 126, 2971

Williams, J. P., \& Cieza, L. A. 2011, ARA\&A, 49, 67

Wright, E. L., Eisenhardt, P. R. M., Mainzer, A. K., et al. 2010, AJ, 140, 1868

Wu, C.-J., Wu, H., Lam, M.-I., et al. 2013, ApJS, 208, 29

Zuckerman, B. 2015, ApJ, 798, 86

Zuckerman, B., Melis, C., Rhee, J. H., Schneider, A., \& Song, I. 2012, ApJ, 752,58 